\title{
Maintenance Optimization for Systems with Multi-dimensional Degradation and Imperfect Inspections
}

\author{
Bin Liu ${ }^{1}$, Xiujie Zhao*2, Yiqi Liu ${ }^{3}$, and Phuc Do ${ }^{4}$ \\ ${ }^{1}$ Department of Management Science, University of Strathclyde, Glasgow, UK \\ ${ }^{2}$ College of Management and Economics, Tianjin University, Tianjin, China \\ ${ }^{3}$ School of Automation Science and Engineering, South China University of Technology, Guangzhou, \\ China \\ ${ }^{4}$ Lorraine University, CRAN, CNRS UMR 7039, Vandoeuvre, 54506, France
}

\begin{abstract}
In this paper, we develop a maintenance model for systems subjected to multiple correlated degradation processes, where a multivariate stochastic process is used to model the degradation processes, and the covariance matrix is employed to describe the interactions among the processes. The system is considered failed when any of its degradation features hits the pre-specified threshold. Due to the dormancy of degradation-based failures, inspection is implemented to detect the hidden failures. The failed systems are replaced upon inspection. We assume an imperfect inspection, in such a way that a failure can only be detected with a specific probability. Based on the degradation processes, system reliability is evaluated to serve as the foundation, followed by a maintenance model to reduce the economic losses. We provide theoretical boundaries of the cost-optimal inspection intervals, which are then integrated into the optimization algorithm to relieve the computational burden. Finally, a fatigue crack propagation process is employed as an example to illustrate the effectiveness and robustness of the developed maintenance policy. The influence of degradation dependence and inspection accuracy is investigated to gain more managerial insights. Numerical results imply that the inspection inaccuracy contributes significantly to the operating cost and it is suggested that more effort should be paid to improve the inspection accuracy.
\end{abstract}

\footnotetext{
${ }^{*}$ Corresponding author. E-mail: xiujizhao2-c@my.cityu.edu.hk
} 
KEY WORDS: Imperfect inspection, multi-dimensional degradation processes, maintenance optimization, hidden failure, cost analysis

\section{Introduction}

Maintenance operation is an effective tool to improve the production quality and reduce economic losses due to system failures. Traditionally, failure data are usually used to establish reliability models, based on which maintenance decisions are made (Zhao et al., 2018; Vu et al., 2018; Wu et al., 2016; Finkelstein et al., 2016; Gao et al., 2019; Qin and Li, 2020). Recently, however, as a result of the improved quality and reliability of systems, it is becoming increasingly more difficult to obtain the failure data, which frustrates failure-model based reliability analysis and the associated maintenance strategies.

As an alternative, degradation-based models have attracted an increasing attention on reliability analysis and maintenance modeling for systems with high reliability. Degradation-based models are established by use of the degradation measurements, which are monitored during system operation (Alaswad and Xiang, 2017; Do et al., 2015; Mo and Xie, 2015). In addition, the degradation-based models are able to characterize the physical failure mechanism and investigate the influence of environmental variations on system degradation processes (Cherkaoui et al., 2018; Deloux et al., 2016; Xu et al., 2019). Degradation-based models have been widely employed to analyze reliability of real systems, e.g., micro-electromechanical systems (MEMS) (Song et al., 2014; Skima et al., 2016), hard disk devices (Ye et al., 2013), and light-emitting diodes (LEDs) (Peng and Tseng, 2009).

In literature, considerable studies have been conducted on degradation-based maintenance (e.g., (Huynh et al., 2012; Xiang et al., 2014; Peng et al., 2012; Liu et al., 2017a,b; Rivera-Gómez et al., 2018; Khatab et al., 2018, 2019)). An implicit assumption of most existant studies is that the system is only suffering one degradation process. However, in reality, as industrial systems are becoming increasingly more complex to perform multiple functions, they are likely to suffer from multi-dimensional degradation processes. Studies on maintenance policies with multiple degradation processes are still very limited. Barker and Newby (2009) proposed a non-periodic inspection strategy for systems with a multivariate degradation process. Liu et al. (2013) develope$\mathrm{d}$ a maintenance strategy for a continuously monitored system subjected to multiple degradation

processes. Liu et al. (2014) developed a preventive maintenance strategy for multi-component systems subjected to degradation, where importance measure was used to determine the critical components. Khatab et al. (2018) established a condition-based selective maintenance model for a 
multi-component system under the constraint of limited duration of breaks between two missions and the required reliability target for the next mission. However, in all studies mentioned above, the degradation processes are assumed to be independent.

In practice, degradation processes are often mutually correlated in the sense that one degradation process affects other degradation processes (Wang and Pham, 2012; Caballé et al., 2015; Keizer et al., 2017; Ma et al., 2018; Qin and Li, 2019). For example, multiple cracks may exist in a rail track, where the degradation processes are imposed upon each crack. Correlation exists among the cracks when the cracks are exposed to common shocks, e.g., traffic load and environmental variations. However, research focusing on maintenance optimization for multiple correlated degradation processes is still lacking. In the literature, there exist several investigations for multicomponent systems where the degradation processes of components are correlated. Hong et al. (2014) developed a condition-based maintenance for a multi-component system with dependent stochastic degradation processes, in which copula was employed to describe the dependence between the degradation processes of components. Lin et al. (2015) proposed importance measures for a multi-component system subjected to dependent degradation process and condition-based maintenance. Rasmekomen and Parlikad (2016) developed a condition-based maintenance for a multi-component system subjected to degradation state-rate interactions. Shen et al. (2018) analyzed system reliability for a multi-component system with interacting components, where the degradation behavior of one component exerts an influence on that of another component. Our study investigates maintenance optimization for system subjected to multi-dimensional degradation processes. Compared with the work focusing on multi-component system with dependent degradation processes between components, mathematically they share some commonalities. Both the systems can be modelled by multiple dependent or independent degradations. The maintenance models applied in a multi-component system can be extended to deal with the multi-dimensional degradation problem. However, the systems differ in nature. We consider a single-unit system that suffers multiple degradations, instead of a multi-component system. Therefore, maintenance on the system will influence all the degradation processes, while for a multi-component system, maintenance on one component may not necessarily affect the degradation processes of other components.

Actually, the multi-degradation phenomena have been reported in various industries, such as in the marine and offshore industry (Von der Ohe et al., 2012). For example, the cylinder position rod is exposed to wear and corrosion in an offshore corrosive environment. The multi-degradation effect between wear and corrosion has been well recognized and referred to as tribocorrosion. The premature surface failure of cylinder position rod is caused by the combined degradation of corrosion, abrasive wear and mechanical tensile loading. 
Degradation-induced failure is referred to as hidden failure or soft failure, which is usually not self-evident and needs to be detected by inspections. An implicit assumption of the existent studies is that the inspection is perfect, i.e., soft failures can always be revealed at inspections (Ben Mabrouk et al., 2020). However, in reality, an inspection may fail to spot the hidden failures due to the limitation of inspection techniques, especially in the presence of harsh environment (Berrade et al., 2013; Chun, 2016; Liu et al., 2016). The probability that a soft failure can be revealed depends on the dedicated inspection techniques. For example, when detecting a crack in a bridge or a rail track, the probability of a crack being detected varies with different inspection techniques, e.g., ultrasonic testing, visual inspection and magnetic particle inspection (Zerbst et al., 2005).

In literature, extensive research has been conducted regarding imperfect inspection (Kallen and van Noortwijk, 2005; Yang and Cho, 2014; He et al., 2015; Sarkar and Saren, 2016; Ye et al., 2019; Liu et al., 2020). He et al. (2015) developed a preventive maintenance scheduling policy based on an imperfect inspection policy, where the authors analytically established the condition for an optimal inspection interval and number of imperfect inspections between two preventive maintenance actions. Levitin et al. (2019) developed an imperfect inspection policy for a mission-oriented system; an optimal inspection interval was obtained to maximize the mission success probability. Cavalcante et al. (2019) investigated the effect of imperfect inspection on a system described by a delay-time model, in which a failure or a defective state was detected with a specific probability. Zhang et al. (2020) proposed a novel maintenance model considering imperfect inspection and imperfect repair, where the defects are detected with a non-constant probability. However, an extensive review of the existing literature reveals that imperfect inspections are investigated largely for systems with a single degradation process, while no research so far has considered imperfect inspection for a system with multi-dimensional degradation processes.

It should be noted that correlation between the degradation processes will influence the chance that system failures are detected. A stronger correlation between the marginal wear processes will imply a smaller chance of a false negative, since a negative inspection has to be the outcome of each dimension of the inspection. Degradation processes with a stronger correlation are more likely to fail together, which increases the probability system failures being detected.

To the best of the authors' knowledge, investigation on multi-dimensional degradation processes under imperfect inspection has not been covered in the maintenance literature. Our research aims to close the gap and contribute to the knowledge of maintenance policy on multiple dependent degradation processes. To this end, in this paper, we develop a maintenance strategy with consideration of imperfect inspection for systems subjected to multi-dimensional degradation processes. A multivariate Wiener process is employed to model the multi-dimensional degradation 
processes, where a covariance matrix characterizes dependency among the degradation processes. The system fails when any of the degradation features exceeds its pre-specified threshold. Periodic inspection is implemented to detect the existence of soft failures. The inspection is imperfect, where a hidden failure is detected with a specific probability. When a hidden failure is detected at inspection, replacement will be carried out immediately to restore the system. Subsequently, a cost model is developed to decide the optimal inspection interval.

We summarize the contribution of this study in the following three aspects.

- We develop a maintenance policy for systems with multi-dimensional degradation processes and investigate the impact of degradation dependence on the maintenance decisions.

- We incorporate the imperfect inspection into the maintenance model and show that the maintenance cost is highly dependent on the inspection accuracy.

- We theoretically determine the range of the maintenance cost and the optimal inspection interval to facilitate optimization.

The remainder of this paper is organized as follows. Section 2 describes the general assumptions of the paper, the degradation modeling and reliability evaluation. Section 3 presents the proposed maintenance policy, with the objective to determine the optimal maintenance cost. In addition, the boundaries of the cost rate and the optimal inspection interval are theoretically investigated. Section 4 presents an example of fatigue crack development to illustrate the degradation process and the advantages of the developed maintenance model. Finally, conclusions and future research directions are summarized in Section 5.

\section{Nomenclature}

$\begin{array}{ll}C R(T) & \begin{array}{l}\text { Long-run cost rate } \\ H_{i}\end{array} \\ K_{1} & \begin{array}{l}\text { Failure threshold of the } i \text { th degradation process } \\ \text { Number of inspections before failure within a }\end{array} \\ & \begin{array}{l}\text { renewal cycle } \\ K_{2}\end{array} \\ m & \begin{array}{l}\text { Number of inspections after failure within a } \\ \text { renewal cycle }\end{array} \\ P_{d}(t) \quad \begin{array}{l}\text { Number of degradation processes } \\ \text { grobability that a failure is revealed at inspection }\end{array} \\ N_{f}(t) \quad \begin{array}{l}\text { Number of failures by time } t\end{array}\end{array}$




$\begin{array}{ll}R(t) & \text { System reliability at time } t \\ T & \text { Inspection interval } \\ S & \text { Length of a renewal cycle } \\ C & \text { Cost in a renewal cycle } \\ T_{d} & \text { System downtime } \\ T_{f} & \text { System failure time } \\ C_{I}, C_{R} & \text { Cost of inspection and replacement } \\ C_{d} & \text { Downtime cost per unit time } \\ U_{j} & \text { Failure set containing } j \text { failures } \\ N_{l} & \text { Number of simulation histories } \\ \mathbf{V}(t) & \mathbf{V}(t)=\left(V_{1}(t), V_{2}(t), \ldots, V_{m}(t)\right)^{T}, m \text {-dimensional } \\ & \text { Brownian motion } \\ \mathbf{X}(t) & \mathbf{X}(t)=\left(X_{1}(t), X_{2}(t), \ldots, X_{m}(t)\right)^{T}, \text { vector of } \\ & \text { degradation values } \\ \beta & \text { Probability that a failure is not detected at inspection } \\ \lambda & \lambda=\left(\lambda_{1}, \lambda_{2}, \ldots, \lambda_{m}\right)^{T}, \text { vector of drift coefficients } \\ \Xi & \text { Variance-covariance matrix } \\ \tau & \text { Parameter of time transformation }\end{array}$

\section{System assumptions and deterioration modelling}

\subsection{System description and assumptions}

Consider a deteriorating system with multi-dimensional degradation processes, in which the degradation processes are indicative of an identical failure mode, e.g., multiple cracks in a rail track. We consider the case that degradation processes only lead to soft failures, that the system is still operating when the degradation level exceeds the failure threshold, but at a higher risk or cost. Hard failure is not considered in this study. The following assumptions are further considered in this paper:

1. The duration of inspection and replacement is negligible when compared with the operating horizon.

2. Inspection has no impact on system degradation.

3. The probability that a failure is discovered at inspection is independent of the existence of other failures. 
4. The probability of failure detection is identical for all the degradation-induced failures.

5. The system is non-repairable; once a failure is detected at inspection, replacement is carried out immediately.

The aforementioned assumptions have been widely used in literature, such as ( $\mathrm{Li}$ and Pham, 2005; Peng et al., 2014). Consider a system that undergoes multiple degradation processes, modeled as a multivariate Wiener process. Wiener process is employed here due to the fact that in reality some systems do not exhibit monotonic degradation behaviors (Zhai and Ye, 2017). For example, the length of a crack in tracks is influenced by various factors, such as temperature, humidity, and track smoothness. The size of cracks evolves non-monotonically. Therefore, we use Wiener process instead of other monotone degradation processes like Gamma and inverse Gaussian process.

Since our work is motivated by the cracks in rail tracks, where multiple cracks may degrade in a track, we only consider soft failures, which occurs when one or more degradation features exceed the failure thresholds, while hard failures are not considered in the study. Definition of system failure in this work is similar to the concept of competing failure modes. It is important to note that after a soft failure occurs, the system continues to operate, but at an unsatisfactory performance level. The system is not continuously monitored, and existence of soft failure is only detected by at periodic inspection (such as a leak in a pipe). For some systems, failure is not self-evident and will have to be detected by inspection, such as cracks in tracks or in bridges. For example, sometimes the crack occurs inside the track or bridge, which is dormant and cannot be discovered by manual vision. Inspection is used for failure detection. Another typical system with latent failures is stand-by redundant systems and protection systems.

Inspection policies are typically classified as periodic inspections and non-periodic inspections. Generally speaking, non-periodic inspection performs better than the periodic inspection policy. However, in practice, non-periodic inspection is not so easy to implement as one needs to rearrange the maintenance staff and reallocate the resources frequently. Therefore, periodic inspection policy is most widely used in real applications. In the literature, there are plenty of studies investigating maintenance polices with periodic inspection.

We assume in this paper that the probability of discovering a failure at inspection is independent of the existence of other failures. That is, given the occurrence of $j$ failures, the probability that at least one failure can be discovered at inspection is $1-\beta^{j}$, where $\beta$ is the probability that an inspection fails to detect a single failure. Usually $\beta$ is determined by the inspection techniques or the inspection cost invested.

After a soft failure occurs (the limit has been reached by one of the variables), the system is in the failed state by this failure mode (damage has been done), regardless of the subsequent 

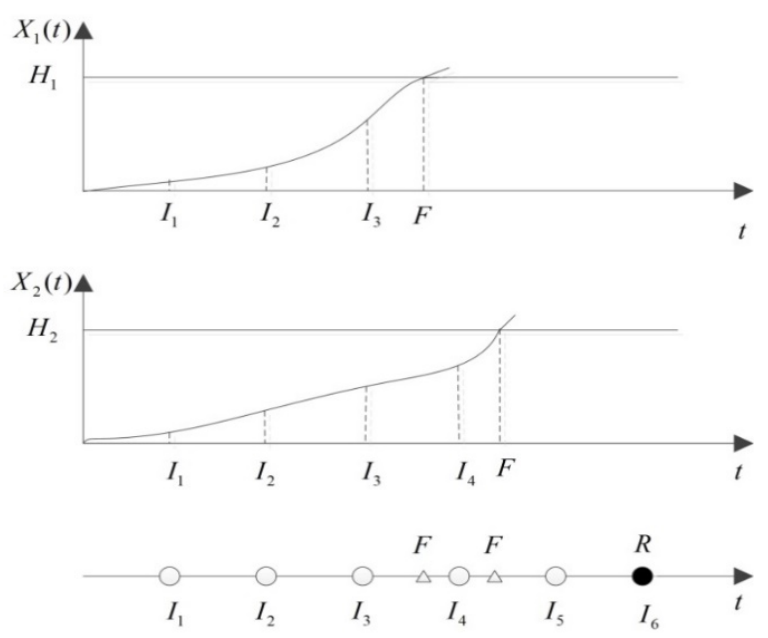

Figure 1: Description of the degradation and inspection process

behaviour of the degradation process. An inspection detects the existing state of the failure mode (with false negative results), and not the degradation process value. Therefore, for each degradation process, it is assumed that inspection simply tells whether a failure occurs or not.

The inspection is imperfect in that a failure can only be detected by a specific probability, which is related to the inspection techniques. We assume a constant probability of failure detection mainly for two reasons. One is that our focus is to develop a maintenance policy for a system with multi-dimensional degradation processes. Inspection accuracy is a realistic factor that influences the maintenance decisions. As the maintenance model itself is quite complicated, considering a varying detection probability will further complicate the model and impede obtaining useful managerial insights. Another reason is that it is difficult to accurately describe the relationship between inspection accuracy and the degradation level. It can be anticipated that probability of failure detection increases with the growth of crack size. However, it is unknown in what form that the two are related, whether in linear, exponential or other forms. Therefore, we assume a constant probability of failure detection to make it simple and focus on the maintenance model. But our model can be extended considering a varying probability of failure detection, at the cost of more computational burden.

Fig. 1 describes the degradation process and inspection schedule for a bivariate case. As presented in Fig. 1, two degradation processes are imposed upon the system, denoted as $X_{1}(t)$ and $X_{2}(t)$. The degradation-induced failures occur after the third and fourth inspection respectively. Imperfect inspection is carried out to detect the occurrence of failures. The failures remain unrevealed until the sixth inspection, where corrective replacement is implemented to restore the system back to a perfect state. 


\subsection{Deterioration process}

Suppose that the system comprises $m$ degradation processes, modeled by a multivariate Wiener degradation process, $\mathbf{X}(t)=\lambda t+\mathbf{V}(t)$, where $\mathbf{X}(t)=\left(X_{1}(t), X_{2}(t), \ldots, X_{m}(t)\right)^{T}$ is the vector of degradation by time $t, \lambda=\left(\lambda_{1}, \lambda_{2}, \ldots, \lambda_{m}\right)^{T}$ denotes the vector of drift coefficients and $\mathbf{V}(t)$ is an $m$ dimensional Brownian motion, $\mathbf{V}(t)=\left(V_{1}(t), V_{2}(t), \ldots, V_{m}(t)\right)^{T}$, characterizing the stochasticity of the degradation processes. In particular, $\mathbf{V}(t)$ follows a multivariate normal distribution, $\mathbf{V}(t) \sim$ $\mathscr{N}(\mathbf{0}, t \Xi)$, where $\Xi$ is the variance-covariance matrix, denoted as $\Xi=\left[\sigma_{i j}\right]$, for $i, j \in\{1, \ldots, m\}$. The joint probability density function (pdf) of $\mathbf{V}(t)$ is expressed as

$$
f\left(v_{1}(t), \ldots, v_{m}(t)\right)=\left(\frac{1}{2 \pi}\right)^{\frac{m}{2}}|t \Sigma|^{-\frac{1}{2}} \exp \left[-\frac{1}{2 t} \mathbf{v}(t)^{T} \Xi^{-1} \mathbf{v}(t)\right] .
$$

According to the degradation process, we have

$$
V_{i}(t)=X_{i}(t)-\lambda_{i} t
$$

The system fails when any of the degradation features $X_{i}$ hits the critical failure threshold $H_{i}$ for the first time, referred to as the first-passage-time. Let $T_{f}$ be the time to failure. It follows

$$
T_{f}=\inf \left\{t: X_{i}(t)>H_{i}, \exists i=1,2, \ldots, m\right\} .
$$

System reliability is given as $R(t)=P\left\{t \leq T_{f}\right\}$. However, there exists no closed-form expression of the FTP for a multi-dimensional Wiener degradation process. Hence, an empirical distribution will be employed to describe the system reliability $R(t)$. The empirical distribution can be obtained from the simulated sample data. System reliability $R(t)$ is expressed as the proportion of degradation observations at time $t$ from the sample that are less than or equal to the failure threshold.

\section{Maintenance policy and optimization}

This section aims to establish the maintenance model and achieve the optimal inspection interval. Periodic inspection is implemented upon epochs $k T$, where $k \in\{1,2,3, \ldots\}$, and $T$ is the inspection interval.

Since the inspection is imperfect, it is possible that a failure is revealed after multiple inspections. If a failure is discovered at inspection, replacement is carried out instantly. The system is restored to the as-good-as-new state upon each replacement. Since the system is restored to a new state, the degradation processes and inspection policy will start over, which constitutes a regenerative process. 
In the following analysis, we will formulate the the long-run cost rate of the maintenance policy by considering the effect of multi-dimensional degradation processes and the inspection accuracy. A search algorithm is then developed to achieve the optimal inspection interval.

\subsection{Maintenance cost model}

In this study, we aim to determine the optimal inspection interval that minimizes the long-run cost rate. In the current model, the cost items involve inspection $\operatorname{cost} C_{I}$, replacement $\operatorname{cost} C_{R}$ and downtime cost per unit time $C_{d}$. Inspection cost is paid whenever an inspection is applied. Downtime cost is paid when the system operates in the (soft) failed state. The basic idea of cost model construction is to investigate the number of inspections before and after failure separately (Berrade et al., 2012). Since before a failure occurs, the expected number of inspection only depends on the distribution of failure time. However, after a failure occurs, the number of inspections is related to the number of existing failures and the inspection accuracy. The distinction in the inspection number leads to the cost modeling. Consider the number of inspections before and after a failure separately can simplify the maintenance cost modeling.

Let $K_{1}$ denote the number of inspections before occurrence of a failure. The probability that $K_{1}$ takes value in $i(i=0,1,2, \ldots)$ is given as

$$
P\left(K_{1}=i\right)=P\left\{T_{f} \in[i T,(i+1) T]\right\}=R(i T)-R((i+1) T)
$$

The expectation of $K_{1}$ is given as

$$
E\left[K_{1}\right]=\sum_{i=0}^{\infty} i P\left(K_{1}=i\right)=\sum_{i=1}^{\infty} R(i T)
$$

Denote $K_{2}$ as the number of inspections in a renewal cycle after a failure occurs. Evaluation of $K_{2}$ is quite tricky, due to the fact that $K_{2}$ not only depends on $K_{1}$, but also on the number of failures at each inspection. Let $N_{f}(t)$ be the number of existing failures by time $t . N_{f}(t)$ takes values in $j$ $(j=0,1, \ldots, m)$ with the probabilities

$$
P\left(N_{f}(t)=j\right)=\sum_{U_{j} \subset\{1, \ldots, m\}:\left|U_{j}\right|=j} P\left\{\begin{array}{l}
\left\{T_{k} \leq t, \forall k \in U_{j}\right\} \\
\cap\left\{T_{l} \leq t, \forall l \notin U_{j}\right\}
\end{array}\right\}
$$

where $U_{j}$ is the set containing $j$ failures, $T_{k}$ and $T_{l}$ are the first-passage-time of the $k$ th and $l$ th degradation processes, i.e., $T_{k}=\inf \left\{t: X_{k}(t)>H_{k}\right\}$ and $T_{l}=\inf \left\{t: X_{l}(t)>H_{l}\right\}$.

Eq. (6) measures all the scenarios that $j$-out-of- $m$ failures have occurred. When $m$ is large, it is tedious to compute all the $P\left(N_{f}(t)=j\right)$, as it contains $2^{m}-1$ subsets. If a system comprises 
a large number of degradation processes (i.e., $m$ is too large), simplification can be achieved by adopting ranking methods such as importance measures to determine the most influential failure modes. For a small $m, P\left(N_{f}(t)=j\right)$ can be calculated via enumeration. Given the occurrence of failures, the probability that at least one failure can be discovered at inspection is given by

$$
P_{d}\left(t \mid t>T_{f}\right)=\sum_{j=1}^{m} P\left(N_{f}(t)=j \mid t>T_{f}\right) \cdot\left(1-\beta^{j}\right) .
$$

For notational simplicity, $P_{d}\left(t \mid t>T_{f}\right)$ is denoted as $P_{d}(t)$. If no failure occurs till time $t$, by definition $P_{d}(t)=0$.

Intuitively, $K_{2}$ is related to $K_{1}$ in a way that a larger $K_{1}$ leads to a more deteriorated system at the time of failure occurrence. Given $K_{1}=k_{1}$, the probability that $K_{2}$ equals to $i$ is given as

$$
\begin{aligned}
& P\left(K_{2}=i \mid K_{1}=k_{1}\right)=P\left\{\bar{A}_{k_{1}+1} \bar{A}_{k_{1}+2} \ldots \bar{A}_{k_{1}+i-1} A_{k_{1}+i} \mid K_{1}=k_{1}\right\} \\
& =\frac{P\left\{\bar{A}_{k_{1}+1} \bar{A}_{k_{1}+2} \ldots \bar{A}_{k_{1}+i-1} A_{k_{1}+i} \cap K_{1}=k_{1}\right\}}{P\left\{K_{1}=k_{1}\right\}} \\
& =\frac{P_{d}\left(k_{1} T+i T\right) \prod_{j=0}^{i-1}\left(1-P_{d}\left(k_{1} T+j T\right)\right)}{R\left(k_{1} T\right)-R\left(\left(k_{1}+1\right) T\right)}
\end{aligned}
$$

where $A_{\left(k_{1}+i\right)}$ denotes the event that a failure is revealed at the $\left(k_{1}+i\right)$ th inspection. Note that based on the definition of $P_{d}(t)$ in Eq. (7), we have $P_{d}\left(k_{1} T\right)=0$ for $k_{1} T<T_{f}$. Eq. (8) denotes the probability that the failure is not discovered until the $\left(k_{1}+i\right)$ th inspection. The probability that $K_{2}$ is no less than $i$ is given as

$$
P\left(K_{2} \geq i\right)=\sum_{k_{1}=0}^{\infty} \prod_{j=0}^{i-1}\left(1-P_{d}\left(k_{1} T+j T\right)\right)
$$

Then the expected $K_{2}$ follows as

$$
\begin{aligned}
E\left[K_{2}\right] & =\sum_{i=1}^{\infty} i P\left(K_{2}=i\right) \\
& =\sum_{i=1}^{\infty} i\left(P\left(K_{2} \geq i\right)-P\left(K_{2} \geq i+1\right)\right)
\end{aligned}
$$

As detection of failures and system replacement can only be carried out at inspections, the length of a renewal cycle is an integer multiple of the inspection interval. The expected length of a renewal cycle can be obtained as

$$
E[S]=\left\{E\left[K_{1}\right]+E\left[K_{2}\right]\right\} T
$$


Let $T_{d}$ be the system downtime, so that $T_{d}=S-T_{f}$. Since it is widely known that

$$
E\left[T_{f}\right]=\int_{0}^{\infty} t d F(t)=-\int_{0}^{\infty} t d R(t)=\int_{0}^{\infty} R(t) d t
$$

we have

$$
E\left[T_{d}\right]=E[S]-E\left[T_{f}\right]=\int_{0}^{\infty} t d R(t)+\left\{E\left[K_{1}\right]+E\left[K_{2}\right]\right\} T
$$

Remark: For a small inspection interval $T$, the expected downtime $E\left[T_{d}\right]$ approaches the time interval of after-failure inspection $E\left[K_{2}\right] T, E\left[T_{d}\right] \approx E\left[K_{2}\right] T$, i.e., $E\left[T_{d}\right]-E\left[K_{2}\right] T \rightarrow 0$ as $T \rightarrow 0$. This is due to $\lim _{T \rightarrow 0} \sum_{i=1}^{\infty} R(i T) T=\int_{0}^{\infty} R(t) d t=-\int_{0}^{\infty} t d R(t)$.

We can have the expected cost within a renewal cycle as

$$
\begin{aligned}
& E[C]=C_{R}+C_{I} \cdot E\left[K_{1}+K_{2}\right]+C_{d} \cdot E\left[T_{d}\right] \\
& =\left(C_{I}+C_{d} T\right) \cdot E\left[K_{1}+K_{2}\right]+C_{R}-C_{d} \cdot E\left[T_{f}\right]
\end{aligned}
$$

Combining Eq. (11) and Eq. (13), the expected long-run cost rate is formulated as

$$
\begin{aligned}
& C R(T)=\lim _{t \rightarrow \infty} \frac{C(t)}{t}=\frac{E[C]}{E[S]} \\
& =C_{d}+\frac{C_{I}}{T}+\frac{C_{R}-C_{d} E\left[T_{f}\right]}{E\left[K_{1}+K_{2}\right] \cdot T}
\end{aligned}
$$

where $C(t)$ is the cumulative cost over the interval $(0, t]$. As presented in Eq. (6), calculation of the probability of number of failures has to take into account all the scenarios whether a degradation process leads to a failure, which is exponentially explosive and computationally prohibitive. Therefore, we fail to achieve a closed-form expression of Eq. (14) for a large number of degradation processes.

Actually, the cost model of Eq. (14) is a generalization of the existing maintenance models. For example, if the inspection is perfect, then the cost model is reduced to a simple inspectionreplacement model (Zhao et al., 2010). This can be simply achieved by setting $P_{d}\left(t \mid t>T_{f}\right)=1$ and $K_{2}=1$. It follows

$$
E[S]=\left(\sum_{i=1}^{\infty} R(i T)+1\right) T
$$


and

$$
\begin{aligned}
& E[C]=C_{R}+C_{I} \cdot\left(\sum_{i=1}^{\infty} R(i T)+1\right) \\
& +C_{d} \cdot\left(T \sum_{i=1}^{\infty} R(i T)+T-E\left[T_{f}\right]\right)
\end{aligned}
$$

Combining the above two equations, we have

$$
C R(T)=C_{d}+\frac{C_{I}}{T}+\frac{C_{R}-C_{d} E\left[T_{f}\right]}{\left(\sum_{i=1}^{\infty} R(i T)+1\right) T}
$$

On the other hand, if one considers a single degradation process, the problem is then reduced to the issue of optimal replacement under imperfect inspection. The number of inspections after failure, $K_{2}$, follows a geometric distribution with parameter $1-\beta$ (Badia et al., 2001). Thus, we have $E\left[K_{2}\right]=1 /(1-\beta)$ and

$$
C R(T)=C_{d}+\frac{C_{I}}{T}+\frac{C_{R}-C_{d} E\left[T_{f}\right]}{\left(\sum_{i=1}^{\infty} R(i T)+\frac{1}{1-\beta}\right) T}
$$

\subsection{Optimization of maintenance cost}

The objective of the study is to minimize the long-run cost rate $C R(T)$ by selecting the optimal $T$,

$$
T^{*}=\arg \min _{T} C R(T)
$$

An analytical solution of the optimization problem (15) cannot be obtained due to the complexity of the expression $C R(T)$. Yet since the inspection interval $T$ is the only decision variable, onedirectional search algorithms can be adopted once $C R(T)$ can be computed, whether theoretically or by simulation. However, following the discussion of Eq. (14), a closed-form expression of $C R(T)$ cannot be achieved generally. Therefore, Monte Carlo simulation will be adopted instead to compute $C R(T)$ (Huynh et al., 2012). With a large number of simulation histories $N_{l}$, Eq. (14) can be expressed as

$$
C R(T)=\frac{E[C]}{E[S]}=\lim _{N \rightarrow \infty} \frac{\sum_{n=1}^{N} C^{(n)}}{\sum_{n=1}^{N} S^{(n)}} \simeq \frac{\sum_{n=1}^{N_{l}} C^{(n)}}{\sum_{n=1}^{N_{l}} S^{(n)}}
$$

where $C^{(n)}$ and $S^{(n)}$ are respectively the cost and length of a renewal cycle for the $n$th simulation history. We determine the number of repetitions for Monte Carlo simulation based on the law of 
large numbers (LLN) and central limit theorem (CLT), with normal distribution which gives very accurate estimate of the number of repetition. LLN and CLT also applies to the length of a renewal cycle $S$. Subsequently, we will investigate the properties of the maintenance cost rate and develop a search algorithm to determine the optimal inspection interval $T$ and maintenance $\operatorname{cost} C R(T)$.

Assume $C_{R}<<C_{d} E\left[T_{f}\right]$ and $C_{I}<<C_{d} E\left[T_{f}\right]$. This assumption is reasonable since in reality the losses due to unexpected failures are much higher than that of replacement and inspection (Van Oosterom et al., 2014). We provide some properties of the maintenance cost to facilitate the optimization process. Proposition 1 provides the upper bound and lower bound of the maintenance cost rate.

Proposition 1. For a given inspection interval $T$, the maintenance cost rate is limited within the range

$$
C_{d}+\frac{C_{I}}{T}+\frac{C_{R}-C_{d} E\left[T_{f}\right]}{E\left[T_{f}\right]+\frac{\beta^{m} T}{1-\beta^{m}}}<C R(T)<C_{d}+\frac{C_{I}}{T}+\frac{C_{R}-C_{d} E\left[T_{f}\right]}{E\left[T_{f}\right]+\frac{T}{1-\beta}}
$$

Detailed proof is provided in the Appendix. Proposition 1 determines the boundary of the longrun cost rate $C R$. Next, we proceed to determine the boundary of the optimal inspection interval $T^{*}$. Denote $f_{1}(T)$ as the lower bound of the long-run cost rate,

$$
f_{1}(T)=C_{d}+\frac{C_{I}}{T}+\frac{C_{R}-C_{d} E\left[T_{f}\right]}{E\left[T_{f}\right]+\frac{\beta^{m} T}{1-\beta^{m}}}
$$

and $f_{2}(T)$ the upper bound of the maintenance cost rate,

$$
f_{2}(T)=C_{d}+\frac{C_{I}}{T}+\frac{C_{R}-C_{d} E\left[T_{f}\right]}{E\left[T_{f}\right]+\frac{T}{1-\beta}}
$$

Proposition 2. The optimal inspection interval $T^{*}$ is limited within the range $T^{*} \in\left(T_{a}, T_{b}\right)$, where $T_{a}$ and $T_{b}$ are the roots of the equation $f_{1}(T)=f_{2}^{*}$, and $f_{2}^{*}$ is the minimum of $f_{2}(T)$,

$$
T_{a}, T_{b} \in\left\{T: f_{1}(T)=f_{2}^{*}\right\}
$$

Detailed proof is provided in the Appendix. Proposition 2 provides the lower and upper bound of the optimal inspection interval. Based on Proposition 2, the optimization algorithm can be developed as follows.

Require: parameters of the degradation processes; cost parameters; parameter of inspection accuracy.

Ensure: optimal inspection interval $T^{*}$ and the associated minimum long-run cost rate $C R^{*}$.

1: start from $T=T_{a}$.

2: repeat 


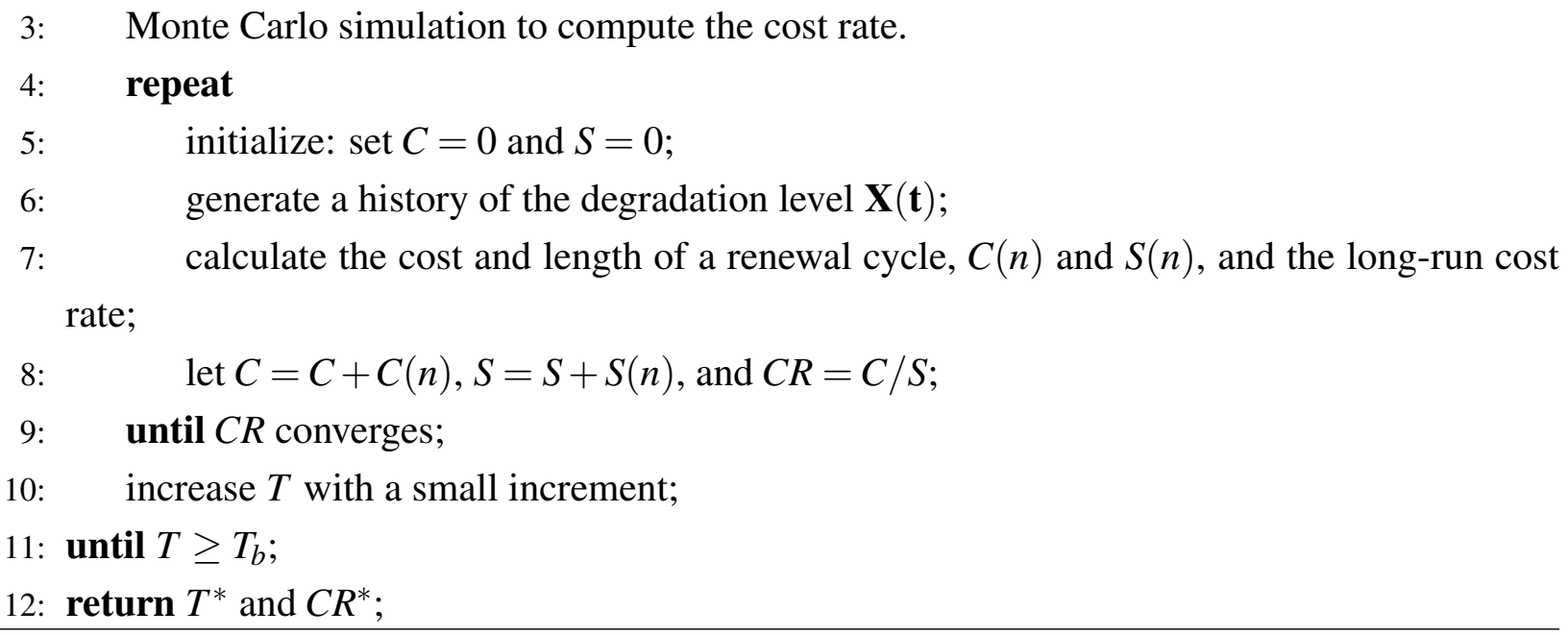

\subsection{Extension: existence of false alarm}

Due to the inaccuracy of inspection techniques, an imperfect inspection not only suffers from the inability to detect a failure but also the possibility of false alarm. False alarm occurs when the detector declares a fault with the system under normal conditions (Berrade et al., 2012). In this section, we will present the impact of false alarm on the maintenance decisions. In presence of false alarm, the system is replaced whenever the detector reports a failed state, either at false alarm or discovery of failure. Based on the type of replacement, the renewal cycle can be divided into two segments: replacement at false alarm or replacement at discovery of failure. Let $E_{1}$ denote the event that replacement occurs at false alarm. The probability that a false alarm occurs before the occurrence of failure is expressed as

$$
\begin{aligned}
P\left(E_{1}\right) & =\alpha \sum_{i=1}^{\infty}(R(i T)-R((i+1) T)) \sum_{j=0}^{i-1}(1-\alpha)^{j} \\
& =\sum_{i=1}^{\infty}(R(i T)-R((i+1) T))\left(1-(1-\alpha)^{i}\right)
\end{aligned}
$$

where $\alpha$ is the probability that a false alarm occurs for a single feature at each inspection. Given that a failure occurs between the $i$ th inspection and the $(i+1)$ th inspection, $T_{f} \in(i T,(i+1) T)$, the conditional probability that a false alarm occurs at the $j$ th inspection is $P_{1, j}=(1-\alpha)^{j-1} \alpha$, for $1 \leq j \leq i$. Since replacement is implemented at false alarm, the expected length of a renewal cycle is given as 


$$
\begin{aligned}
& E\left[S \mid T_{f} \in(i T,(i+1) T), E_{1}\right]=T \sum_{j=1}^{i} j P_{1, i} \\
& =\alpha T \sum_{j=1}^{i} j(1-\alpha)^{j-1} \\
& =\frac{T\left[1-(1+i \alpha)(1-\alpha)^{i}\right]}{\alpha}
\end{aligned}
$$

The expected cost of a renewal cycle is expressed as

$$
E\left[C \mid T_{f} \in(i T,(i+1) T), E_{1}\right]=\frac{C_{I}\left[1-(1+i \alpha)(1-\alpha)^{i}\right]}{\alpha}+C_{R}
$$

Denote $E_{2}$ as the event that the system is replaced at discovery of failure. $E_{2}$ implies that no false alarm occurs before the system fails; the associated probability is denoted as

$$
P\left(E_{2}\right)=\sum_{i=0}^{\infty}(R(i T)-R((i+1) T))(1-\alpha)^{i}
$$

The expected length and cost of a renewal cycle conditioned on $E_{2}$ can be obtained in a similar way. The long-run cost rate can then be formulated by combining the scenarios of $E_{1}$ and $E_{2}$. However, as it is tedious to express an analytical form of $E[C]$ and $E[T]$, we will resort to Monte Carlo to calculate the expected cost and length of a renewal cycle.

\section{An illustrative example}

In this section we use a system with two fatigue crack deterioration processes to illustrate the effectiveness of the proposed maintenance policy. The data of fatigue crack sizes are obtained from Meeker and Escobar (1998), which have been widely used for reliability evaluation and maintenance modeling (e.g., Pan et al. (2013)). The data were collected from fatigue crack growth experiments of the alloy under constant load amplitude duty cycles (Meeker and Escobar, 1998). In the original data, 21 units are tested for fatigue crack propagation and the measurements are observed at the same measurement times. Each unit is recorded per 0.01 million cycles, measured till 0.09 million cycles. To illustrate the multi-dimensional degradation process, we assume that there is a product with two possible fatigue crack positions. 20 units are selected and half of the units are treated as if they are for the first degradation process and the other half for the second degradation process. In other words, measurements of 10 units are used for each degradation 
process (crack propagation). The system is considered failed if any of the two cracks exceeds 1.6 in. in size. In the following we estimate the degradation parameters as first step and then evaluate the maintenance policy.

Remark 4.1 Some of the existing studies were using gamma process for crack propagation. But there are also some reports using Wiener process to describe the crack propagation (e.g., Pan et al. (2013)). Wiener process with a small variance is also capable to describe the degradation data that presents an increasing trend. Actually, our maintenance model is very general that can be easily adapted to a gamma process.

\subsection{Estimation of degradation parameters}

It should be noted that the aforementioned degradation process exhibits a nonlinear behavior (Pan et al., 2013). For cases where the degradation path is not linear, a time scale transformation can be used for linearization (Wang et al., 2014). The transform is denoted as $\tau=\tau(t, \gamma)$. The most commonly used time scale transformations are exponential and power time transformation (Whitmore and Schenkelberg, 1997), i.e., $\tau=1-\exp \left(-\eta t^{\gamma}\right)$ or $\tau=t^{\gamma}$, where $\eta$ and $\gamma$ are positive parameters. With time scale transformation, the corresponding reliability function can be obtained by replacing $t$ with $\tau$. Here we model the deterioration process by a bivariate Wiener process with a power time transform $\tau=t^{\gamma}$. The parameter $\gamma$ is set to be 1.3 as in the work of Pan et al. (2013). The degradation model is fitted with the fatigue crack data and maximum likelihood estimation (MLE) is performed to estimate associated parameters. The estimates are presented in Table 1 , where $\rho$ is the correlation coefficient. Detailed MLE procedure is given in the Appendix.

Table 1: Estimates of the degradation parameters

\begin{tabular}{lllllll}
\hline Parameters & $\lambda_{1}$ & $\lambda_{2}$ & $\sigma_{11}$ & $\sigma_{12}\left(\sigma_{21}\right)$ & $\sigma_{22}$ & $\rho$ \\
\hline Estimates & 12.2187 & 7.5967 & 0.0505 & 0.0147 & 0.0247 & 0.4162 \\
\hline
\end{tabular}

We are interested in the value of $\gamma$, as $\gamma$ dominates the transformation of time scale. Sensitivity analysis is performed to investigate the impact of different $\gamma$ on the estimates of $\lambda$ and $\gamma$. Fig. 2 presents the variability of estimated $\lambda$ in term of different $\gamma$ and Fig. 3 shows the influence on estimated $\sigma$. It can be observed that both the estimates of $\lambda_{1}$ and $\lambda_{2}$ increase with the value of $\gamma$. This is due to the fact that an increased $\gamma$ reduces the transformed interval of two consecutive measurements. Fig. 3 shows an obvious increasing trend of $\gamma$ when $\gamma$ is larger than 1.3. The increasing trend is obvious for a large $\gamma$. 


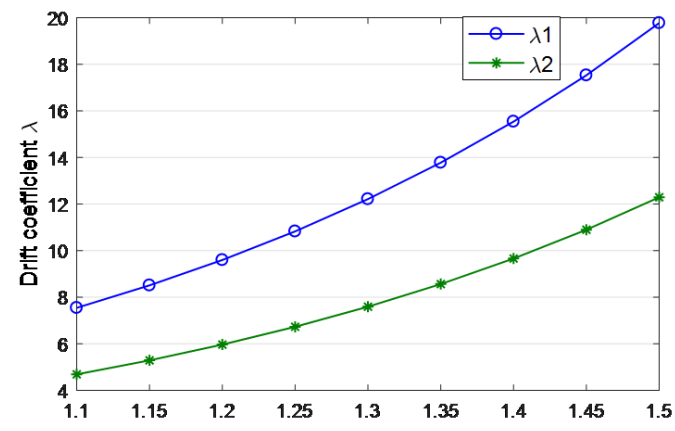

Figure 2: Variability of estimated $\lambda$ with respect to $\gamma$

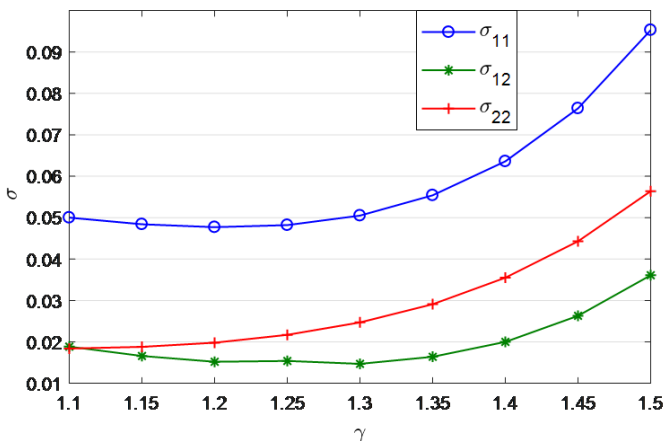

Figure 3: Variability of estimated $\sigma$ with respect to $\gamma$

\subsection{Optimal maintenance policy}

The system is deemed as failed whenever the crack size hits the failure threshold of 1.6in. (Pan et al., 2013). Since the initial degradation levels of the two cracks are 0.9in., to balance the influence of initial degradation, we redefine the failure threshold as $H_{1}=H_{2}=0.7 \mathrm{in}$.. We assume that the spare parts for replacement are collected from the same batch, so that the initial degradation values after replacement are identical to the previous ones. With the estimates of model parameters, system reliability can be numerically evaluated. Fig. 4 plots the system reliability function.

When the volatility of the degradation processes is not too large, system reliability can be approximated as follow

$$
\begin{aligned}
& R(t) \approx \int_{0}^{H_{1}} \ldots \int_{0}^{H_{m}} f\left(x_{1}(t), \ldots, x_{m}(t)\right) d x_{1} \ldots d x_{m} \\
& =\int_{-\infty}^{H_{1}} \ldots \int_{-\infty}^{H_{m}}\left(\frac{1}{2 \pi}\right)^{\frac{m}{2}}|t \Xi|^{-\frac{1}{2}} \exp \left(-\frac{1}{2 t} W(t)\right) d x_{1} \ldots d x_{m},
\end{aligned}
$$




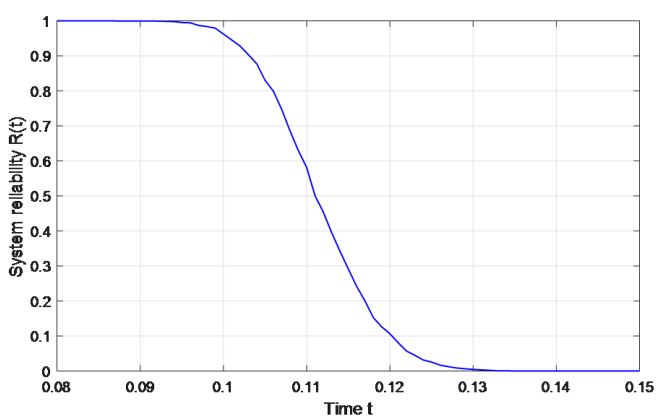

Figure 4: System reliability function

where $W(t)=(\mathbf{x}(t)-\lambda t)^{T} \Xi^{-1}(\mathbf{x}(t)-\lambda t)$.

System reliability of Wiener processes are evaluated using the first-passage-time, i.e., the time epoch when the degradation level hits the threshold for the first time. Generally, the approximation of Eq (16) fails to describe the first-passage-time, since Wiener process is not a monotonic process, that the degradation level may cross the failure threshold and then drop below the threshold at inspection. However, this effect is not significant when the volatility is small (diffusion parameter of the Wiener process is relatively small compared with the drift parameter)(Elwany et al., 2011). To illustrate the appropriateness of the approximation, we compare the original and approximated reliability function in terms of distribution similarity. Fig. 5 presents the comparison under different covariances, where

$$
\Xi=\left[\begin{array}{ll}
\sigma_{11} & \sigma_{12} \\
\sigma_{21} & \sigma_{22}
\end{array}\right]=\left[\begin{array}{ll}
0.0505 & 0.0147 \\
0.0147 & 0.0247
\end{array}\right]
$$

It can be observed from Fig. 5 that the approximation of reliability function is quite effective.

In addition, we employ Hellinger distance to measure the similarity of the original and the approximated reliability function (Beran, 1977; Ciabattoni et al., 2018). Let $F_{o}(t)$ denote the original cumulative distribution function of the failure time $T_{f}$ and $F_{a}(t)$ the approximated one. Hellinger distance is expressed as

$$
\begin{aligned}
H\left(F_{o}, F_{a}\right) & =\frac{1}{2} \int_{0}^{\infty}\left(\sqrt{\frac{d F_{o}(t)}{d t}}-\sqrt{\frac{d F_{a}(t)}{d t}}\right)^{2} d t \\
& =1-\int_{0}^{\infty} \sqrt{\left(\frac{d F_{o}(t)}{d t} \frac{d F_{a}(t)}{d t}\right)} d t
\end{aligned}
$$

Fig. 6 presents the Hellinger distance in terms of the multiplier of covariance matrix. It can be observed that the Hellinger distance increases with the covariance, which indicates that the performance of approximation decreases with the covariance. It can can be concluded the approximation is effective given the present covariance. 

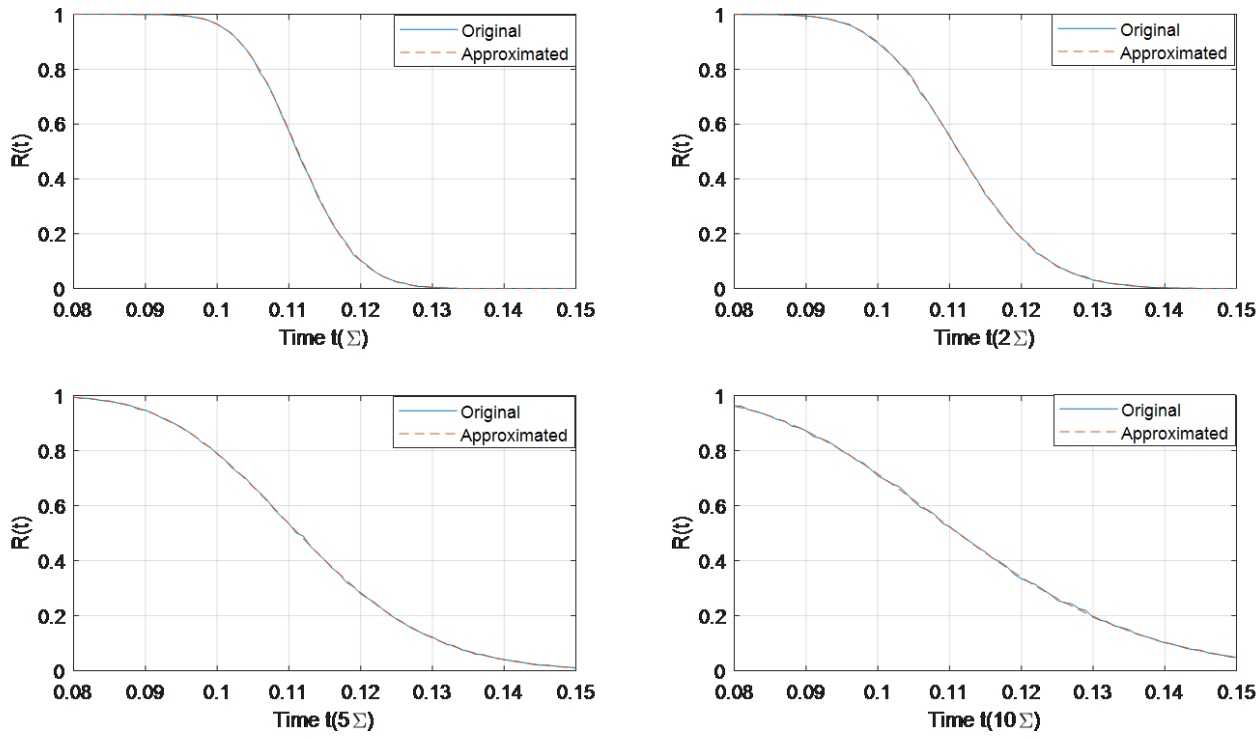

Figure 5: Comparison of system reliability under different covariances

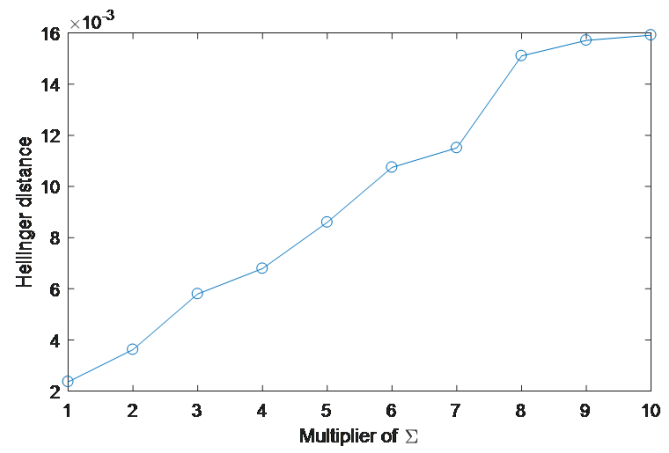

Figure 6: Plot of Hellinger distance

The cost parameters in this paper serve for the purpose of illustration. The values are given as $C_{I}=2, C_{R}=50$ and $C_{d}=2000$. The probability that an inspection fails to detect a failure is dependent on the inspection technique and the type of failure. The inspection inaccuracy $\beta$ is set as $\beta=0.4$. Based on Proposition 2, the lower and upper bounds of the optimal inspection interval can be obtained as $T_{a}=0.0053$ and $T_{b}=0.1997$. In addition, for illustrative purpose, we plot how $f_{1}(t)$ and $f_{2}(t)$ vary with the inspection interval $T$, as shown in Fig. 7.

By searching within the range $\left(T_{a}, T_{b}\right)$, we obtain the minimal long-run cost rate $C R^{*}=675$, and the optimal inspection interval $T^{*}=0.0512$. Fig. 8 shows the variation of maintenance cost rate. By comparison, if the inspection is perfect, i.e., $\beta=0$, the minimal expected cost rate is obtained as $C R^{*}=607$, at $T^{*}=0.103$. The results show a large difference in $T^{*}$ by introducing 


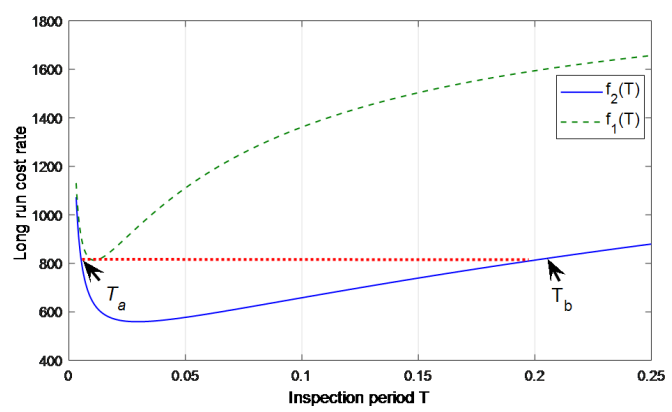

Figure 7: Plot of $f_{1}(t)$ and $f_{2}(t)$

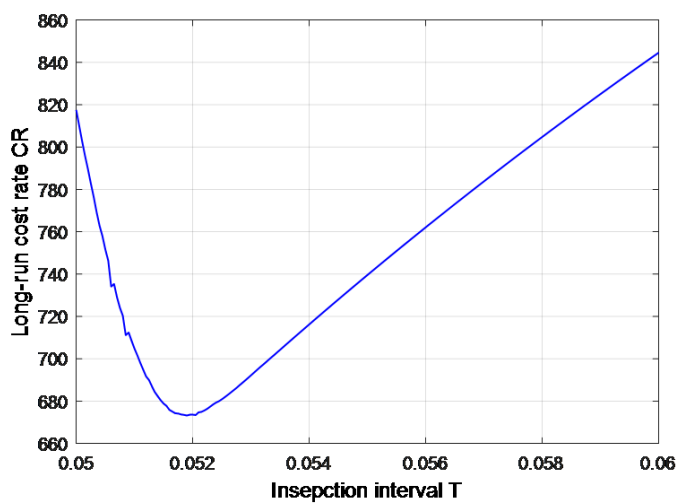

Figure 8: Maintenance cost rate vs inspection intervals
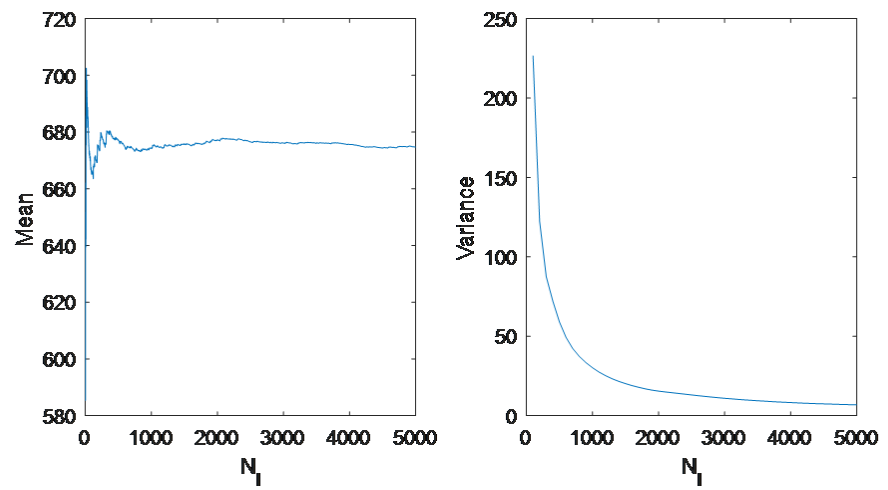

Figure 9: Mean and variance of $C R^{*}$ with number of repetitions

the imperfect inspection assumption. To show the convergence of the maintenance cost rate, we plot how the mean and variance of $C R^{*}$ vary with respect to $N_{l}$ in Fig. 9. It can be observed that the variance decreases with the number of repetitions and the maintenance cost rate gradually converges to $C R^{*}=675$. 


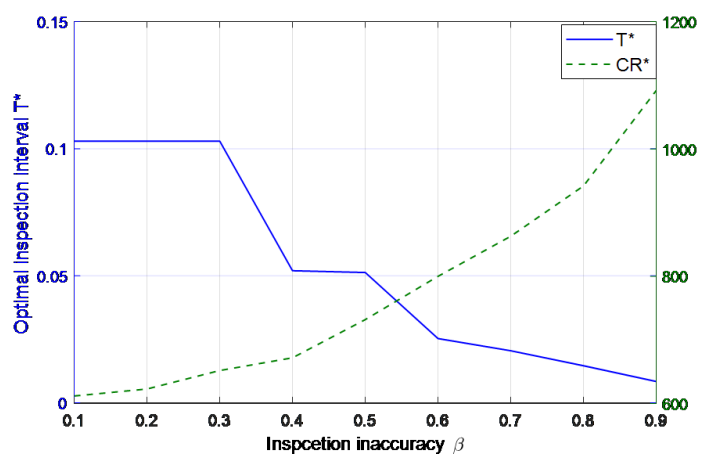

Figure 10: Variability of $C R\left(T^{*}\right)$ and $T^{*}$ vs $\beta$

Sensitivity analysis on $\beta$ is performed to investigate the impact of different inspection techniques on the long-run cost rate. We present in Fig. 10 the variation of $C R\left(T^{*}\right)$ and $T^{*}$ with respect to $\beta$. As can be seen, the minimal cost rate $C R\left(T^{*}\right)$ varies from 612 to 1092 when $\beta$ increases from 0.1 to 0.9 . The positive increasing relationship indicates that the long-run cost rate is highly dependent on the inspection accuracy. It is suggested that managers or engineers should pay more effort to improve the inspection accuracy so as to reduce the loss caused by soft failures. In addition, the optimal inspection interval $T^{*}$ shows a nonincreasing trend with respect to the inspection accuracy $\beta$. This can be explained by the fact that an accurate inspection technique is more likely to detect the failure, which calls for a less frequent inspection policy.

Variation of inspection cost in terms of its accuracy improvement is not considered in this study. However, high inspection accuracy always comes at a price. There exists a tradeoff between improvement of inspection accuracy and its cost. The more accurate, the higher cost an inspection technique will incur. Practitioners may be more interested in the analysis of inspection interval and the corresponding overall cost with different inspection accuracy at different cost. Actually, the result in Fig. 10 can be used to evaluate the effectiveness of a specific inspection technique against its cost. If we have the information regarding inspection accuracy and the associated cost for a specific inspection technique, the optimal inspection technique can be indicated by comparing its effectiveness in maintenance cost reduction against the cost. In addition, we compare with gamma processes to show the applicability of the proposed model. Details are shown in the Appendix.

For illustrative purpose, we consider the case that the company needs to invest in a more advanced device to improve the inspection accuracy. The investment cost in terms of the inspection inaccuracy $\beta$ is given as $300 \times(1-\beta)$. Fig. 11 presents the variability of the optimal maintenance cost considering the additional investment cost. It can be observed that the optimal maintenance cost does not present a monotonic trend considering the additional investment cost. Instead, the minimal maintenance cost is achieved at inspection inaccuracy $\beta=0.4$. 


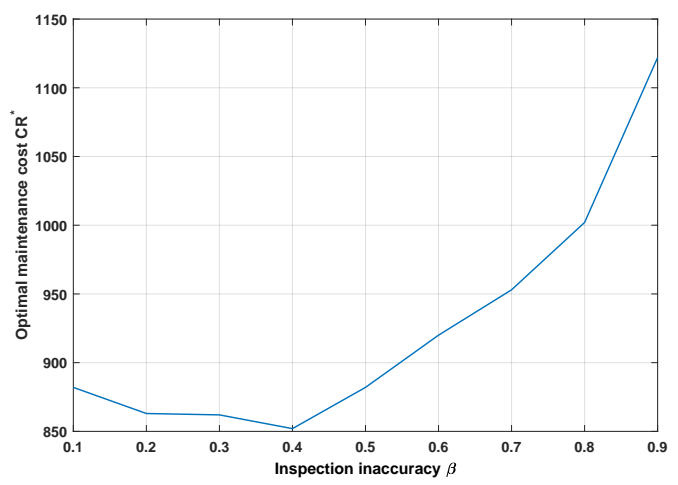

Figure 11: Variability of $C R\left(T^{*}\right)$ considering additional investment cost

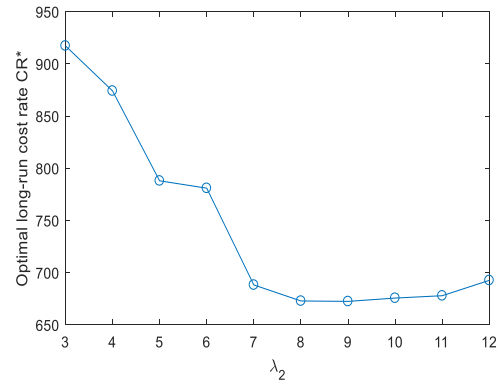

(a)

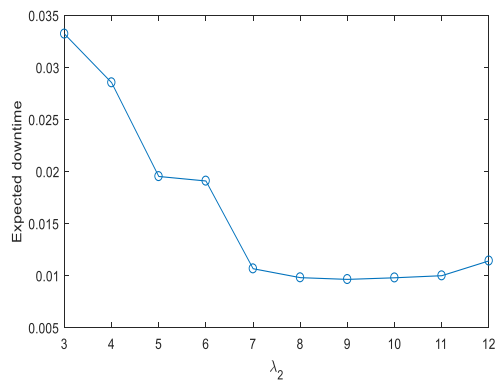

(b)

Figure 12: Variability of (a) $C R^{*}$ and (b) expected downtime with $\lambda_{2}$

In addition, to illustrate the influence of multiple degradation processes, we plot the variation of $C R^{*}$ with respect to $\lambda_{2}$ in Fig. 12. It is interesting to observe that $C R^{*}$ shows a decreasing trend for small $\lambda_{2}$, and and increasing trend for $\lambda_{2} \geq 8$. Intuitively, $C R^{*}$ should increase monotonically with $\lambda_{2}$. We believe this is due to the fact that existence of multiple failures increases the probability of failure detection and reduces the downtime time. For a small $\lambda_{2}$, process 2 is dominated by process 1 , so as if we have one process. When $\lambda_{2}$ increases, it will be as dominant as process 1 , which leads to an increased probability of failure detection and a decreased maintenance cost. But with further increase of $\lambda_{2}$, process 2 becomes dominant, so we are again back to the situation as with one process. To validate this conjecture, we also present how the expected downtime varies with $\lambda_{2}$ in Fig. 12, which shows that the expected downtime exhibits a similar trend as $C R^{*}$.

To compare with the maintenance performance under independent degradation processes, Fig. 13 shows the variation of long-run cost rate with the covariances $\left(\sigma_{12}\right.$ and $\left.\sigma_{21}\right)$ set to 0 . If the degradation processes are independent, the optimal long-run cost rate is achieved as $C R^{*}=678.7$, at the inspection interval $T^{*}=0.0514$. 


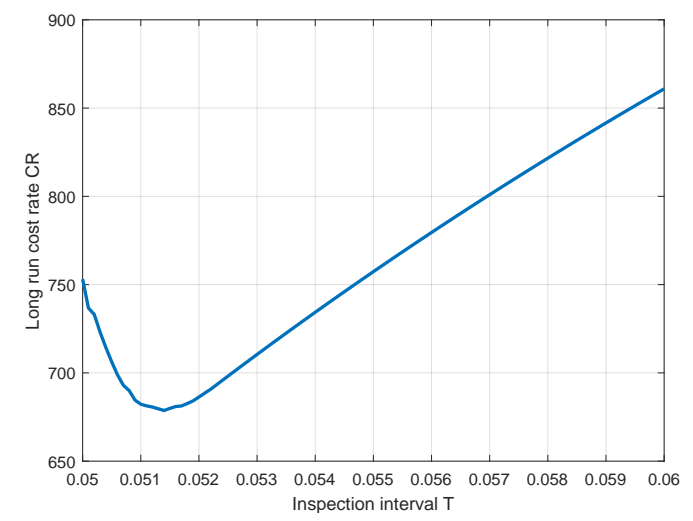

Figure 13: Plot of $C R$ under independent degradation processes

\subsection{Performance analysis of the maintenance policy}

We are interested to investigate the maintenance model under parameter estimation uncertainty with the given data. To study the robustness of the maintenance policy, we employ asymptotic approaches to quantify the parameter estimation uncertainty. Under reasonably large sample sizes, a multivariate normal (MVN) distribution is able to approximate the maximum likelihood estimators (Zheng and Fang, 2018). For convenience, we reparametrize the elements in covariance matrix as: $\sigma_{1}=\sqrt{\sigma_{11}}, \sigma_{2}=\sqrt{\sigma_{22}}$ and $\rho=\sigma_{12} / \sigma_{1} \sigma_{2}$, then the associated unknown parameters is $\theta=\left(\lambda_{1}, \lambda_{2}, \sigma_{1}, \sigma_{2}, \rho\right)^{T}$. Denote $\hat{\boldsymbol{\theta}}$ as the MLE of $\theta$. The asymptotic distribution of $\hat{\theta}$ is approximated by a MVN as $\hat{\theta} \sim \mathscr{N}\left(\hat{\theta},[\mathrm{I}(\hat{\theta})]^{-1}\right)$, where $I(\hat{\theta})$ is the Fisher information matrix at $\hat{\theta}$. Detailed derivation of the Fisher information matrix is provided in the Appendix. The Fisher information matrix can be obtained as

$$
I(\hat{\boldsymbol{\theta}})=\left[\begin{array}{ccccc}
10.5 & 6.23 & 0 & 0 & 0 \\
6.23 & 21.4 & 0 & 0 & 0 \\
0 & 0 & 3928 & -554 & -202 \\
0 & 0 & -554 & 8051 & -288 \\
0 & 0 & -202 & -288 & 154
\end{array}\right]
$$

and the covariance matrix

$$
\operatorname{Cov}(\hat{\boldsymbol{\theta}})=\left[\begin{array}{ccccc}
1156 & -336 & 0 & 0 & 0 \\
-336 & 565 & 0 & 0 & 0 \\
0 & 0 & 2.81 & 0.34 & 4.3 \\
0 & 0 & 0.34 & 1.37 & 3 \\
0 & 0 & 4.3 & 3 & 76
\end{array}\right] \times 10^{-4}
$$



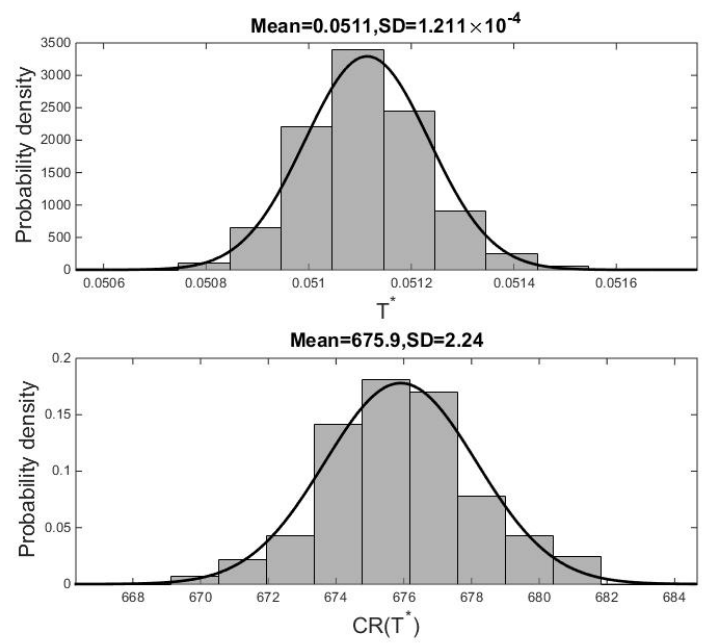

Figure 14: Histogram of $T^{*}$ and $C R\left(T^{*}\right)$, and the associated fitted normal pdf curves

$\hat{\theta} \mid \theta$ converges to $\theta$ in distribution with the increase of sample size, i.e., $\sqrt{n}(\hat{\theta}-\theta) \rightarrow N\left(0,[I(\theta)]^{-1}\right)$, which implies that $\hat{\theta}$ gives a good approximation of $\theta$.

The observed Fisher information can be employed to evaluate the asymptotic distribution of MLE. By drawing from $\mathscr{N}\left(\hat{\boldsymbol{\theta}},[\mathrm{I}(\hat{\boldsymbol{\theta}})]^{\mathbf{- 1}}\right)$, we can obtain the samples that describe the uncertainty of the MLE.

We generate 1000 samples from the distribution $\mathscr{N}\left(\hat{\boldsymbol{\theta}},[\mathrm{I}(\hat{\boldsymbol{\theta}})]^{-\mathbf{1}}\right)$. One sample refers to the set of the estimated parameters, $\theta=\left(\lambda_{1}, \lambda_{2}, \sigma_{1}, \sigma_{2}, \rho\right)^{T}$. For each sample, we obtain the optimal inspection interval $T^{*}$ and the associated minimal long-run cost rate $C R\left(T^{*}\right)$. To investigate how $T^{*}$ and $C R\left(T^{*}\right)$ vary with the uncertain estimates of the parameters, we plot the histogram of $T^{*}$ and $C R\left(T^{*}\right)$ in Fig. 14. As shown in Fig. 14, compared with the mean of $T^{*}$ and $C R\left(T^{*}\right)$, the standard deviation is quite small, which indicates a strong robustness of the maintenance policy.

\section{Conclusions}

This paper presents a maintenance policy for systems subjected to multi-dimensional degradation processes. Imperfect inspection is implemented to detect the dormant failures, where a failure can only be discovered with a certain probability. For the multivariate degradation process, a reliability model is formulated as a first step, followed by a cost model as the objective function. Our study shows that the optimal inspection interval is mostly influenced by the inspection accuracy and engineers or managers are suggested to pay more effort to improving the inspection quality so as to sustain system operation and reduce economic losses. The effect of multiple degradation 
processes is twofold. On one hand, existence of multiple failures can boost the probability of failure detection and thus can reduce downtime cost. On the other hand, a degradation process with a large drift rate may make the system more prone to failure and increase the maintenance cost.

The proposed maintenance model has many potential applications in real life. For example, to detect the crack size in infrastructures, there may exist multiple options such as visual inspection, magnetic particle inspection, and ultrasonic testing. By balancing the benefits and cost of the various testing options, we can determine the most economic inspection technique and the associated inspection frequency. Additionally, in some cases, it is tricky to model all the degradations in presence of numerous degradation processes. Then a decision maker may focus on several most significant degradation processes and develop maintenance policies accordingly.

In the future, the present maintenance model can be extended in the following two directions. First, in the current model, we assume a constant detection probability of the failures. Yet, in reality, the detection probability may depend on the degradation level, in addition to the inspection techniques. It would be more reasonable to model the detection probability as a function of the degradation level and the inspection cost. Second, we assume in the current paper that the downtime cost rate remains constant beyond system failure, regardless of the number of existing failures. In reality, it would be more reasonable to model the downtime cost rate as a function of the number of the existing failures.

\section{Acknowledgements}

The authors would like to thank the associate editor and three anonymous reviewers for their constructive comments that substantially help to improve the paper. This work was supported by the National Natural Science Foundation of China (61873096, 62073145, 71971181, 72002149), Guangdong Basic and Applied Basic Research Foundation (2020A1515011057), and Guangdong Technology International Cooperation Project Application (2020A0505100024).

\section{References}

Suzan Alaswad and Yisha Xiang. A review on condition-based maintenance optimization models for stochastically deteriorating system. Reliability Engineering \& System Safety, 157:54-63, 2017. 
Francisco German Badia, María Dolores Berrade, and Clemente A Campos. Optimization of inspection intervals based on cost. Journal of Applied Probability, 38(4):872-881, 2001.

Colin T Barker and Mark J Newby. Optimal non-periodic inspection for a multivariate degradation model. Reliability Engineering \& System Safety, 94(1):33-43, 2009.

Amel Ben Mabrouk, Anis Chelbi, Lazher Tlili, and Mehdi Radhoui. A quasi-optimal inspection strategy for leased equipment. International Journal of Production Research, 58(3):878-892, 2020.

Rudolf Beran. Minimum hellinger distance estimates for parametric models. The Annals of Statistics, 5(3):445-463, 1977.

María Dolores Berrade, Cristiano AV Cavalcante, and Philip A Scarf. Maintenance scheduling of a protection system subject to imperfect inspection and replacement. European Journal of Operational Research, 218(3):716-725, 2012.

María Dolores Berrade, Philip A Scarf, Cristiano Alexandre Virgínio Cavalcante, and Richard A Dwight. Imperfect inspection and replacement of a system with a defective state: A cost and reliability analysis. Reliability Engineering \& System Safety, 120:80-87, 2013.

Nuria Caballe Caballé, Inma T Castro, Carlos J Pérez, and José Manuel Lanza-Gutiérrez. A condition-based maintenance of a dependent degradation-threshold-shock model in a system with multiple degradation processes. Reliability Engineering \& System Safety, 134:98-109, 2015.

Cristiano AV Cavalcante, Philip A Scarf, and MD Berrade. Imperfect inspection of a system with unrevealed failure and an unrevealed defective state. IEEE Transactions on Reliability, 68(2): 764-775, 2019.

Hajar Cherkaoui, Khac Tuan Huynh, and Antoine Grall. Quantitative assessments of performance and robustness of maintenance policies for stochastically deteriorating production systems. International Journal of Production Research, 56(3):1089-1108, 2018.

Young $\mathrm{H}$ Chun. Improved method of estimating the product quality after multiple inspections. International Journal of Production Research, 54(19):5686-5696, 2016.

Lucio Ciabattoni, Francesco Ferracuti, Alessandro Freddi, and Andrea Monteriu. Statistical spectral analysis for fault diagnosis of rotating machines. IEEE Transactions on Industrial Electronics, 65(5):4301-4310, 2018. 
Estelle Deloux, Mitra Fouladirad, and Christophe Bérenguer. Health-and-usage-based maintenance policies for a partially observable deteriorating system. Proceedings of the Institution of Mechanical Engineers, Part O: Journal of Risk and Reliability, 230(1):120-129, 2016.

Phuc Do, Alexandre Voisin, Eric Levrat, and Benoit Iung. A proactive condition-based maintenance strategy with both perfect and imperfect maintenance actions. Reliability Engineering \& System Safety, 133:22-32, 2015.

Alaa H Elwany, Nagi Z Gebraeel, and Lisa M Maillart. Structured replacement policies for components with complex degradation processes and dedicated sensors. Operations Research, 59 (3):684-695, 2011.

Maxim Finkelstein, Mahmood Shafiee, and Anselme N Kotchap. Classical optimal replacement strategies revisited. IEEE Transactions on Reliability, 65(2):540-546, 2016.

Hongda Gao, Lirong Cui, and Jianhui Chen. Reliability modeling for sparsely connected homogeneous multistate consecutive-k-out-of-n: G systems. IEEE Transactions on Systems, Man, and Cybernetics: Systems, 2019.

Kai He, Lisa M Maillart, and Oleg A Prokopyev. Scheduling preventive maintenance as a function of an imperfect inspection interval. IEEE Transactions on reliability, 64(3):983-997, 2015.

Han-Ping Hong, Wenxing Zhou, Shenwei Zhang, and W Ye. Optimal condition-based maintenance decisions for systems with dependent stochastic degradation of components. Reliability Engineering \& System Safety, 121:276-288, 2014.

Khac Tuan Huynh, Anne Barros, and Christophe Bérenguer. Maintenance decision-making for systems operating under indirect condition monitoring: value of online information and impact of measurement uncertainty. IEEE Transactions on Reliability, 61(2):410-425, 2012.

Maarten-Jan Kallen and Jan M van Noortwijk. Optimal maintenance decisions under imperfect inspection. Reliability engineering \& system safety, 90(2-3):177-185, 2005.

Minou CA Olde Keizer, Simme Douwe P Flapper, and Ruud H Teunter. Condition-based maintenance policies for systems with multiple dependent components: A review. European Journal of Operational Research, 261(2):405-420, 2017.

Abdelhakim Khatab, Claver Diallo, El-Houssaine Aghezzaf, and Uday Venkatadri. Conditionbased selective maintenance for stochastically degrading multi-component systems under periodic inspection and imperfect maintenance. Proceedings of the Institution of Mechanical Engineers, Part O: Journal of Risk and Reliability, 232(4):447-463, 2018. 
Abdelhakim Khatab, Claver Diallo, El-Houssaine Aghezzaf, and Uday Venkatadri. Integrated production quality and condition-based maintenance optimisation for a stochastically deteriorating manufacturing system. International Journal of Production Research, 57(8):2480-2497, 2019.

Gregory Levitin, Maxim Finkelstein, and Hong-Zhong Huang. Scheduling of imperfect inspections for reliability critical systems with shock-driven defects and delayed failures. Reliability Engineering \& System Safety, 189:89-98, 2019.

Wenjian Li and Hoang Pham. An inspection-maintenance model for systems with multiple competing processes. IEEE Transactions on Reliability, 54(2):318-327, 2005.

Yan-Hui Lin, Yan-Fu Li, and Enrico Zio. Component importance measures for components with multiple dependent competing degradation processes and subject to maintenance. IEEE Transactions on Reliability, 65(2):547-557, 2015.

Bin Liu, Zhengguo Xu, Min Xie, and Way Kuo. A value-based preventive maintenance policy for multi-component system with continuously degrading components. Reliability Engineering \& System Safety, 132:83-89, 2014.

Bin Liu, Xiujie Zhao, Ruey-Huei Yeh, and Way Kuo. Imperfect inspection policy for systems with multiple correlated degradation processes. IFAC-PapersOnLine, 49(12):1377-1382, 2016.

Bin Liu, Shaomin Wu, Min Xie, and Way Kuo. A condition-based maintenance policy for degrading systems with age-and state-dependent operating cost. European Journal of Operational Research, 263(3):879-887, 2017a.

Bin Liu, Ruey-Huei Yeh, Min Xie, and Way Kuo. Maintenance scheduling for multicomponent systems with hidden failures. IEEE Transactions on Reliability, 66(4):1280-1292, 2017b.

Bin Liu, Phuc Do, Benoit Iung, and Min Xie. Stochastic filtering approach for condition-based maintenance considering sensor degradation. IEEE Transactions on Automation Science and Engineering, 17(1):177-190, 2020.

Xiao Liu, Jingrui Li, Khalifa N Al-Khalifa, Abdelmagid S Hamouda, David W Coit, and Elsayed A Elsayed. Condition-based maintenance for continuously monitored degrading systems with multiple failure modes. IIE Transactions, 45(4):422-435, 2013.

Zhenzhen Ma, Jianjun Zhu, and Ye Chen. A probabilistic linguistic group decision-making method from a reliability perspective based on evidential reasoning. IEEE Transactions on Systems, Man, and Cybernetics: Systems, (99):1-15, 2018. 
William Q Meeker and Luis A Escobar. Statistical methods for reliability data. John Wiley \& Sons, 1998.

Huadong Mo and Min Xie. A dynamic approach to performance analysis and reliability improvement of control systems with degraded components. IEEE Transactions on Systems, Man, and Cybernetics: Systems, 46(10):1404-1414, 2015.

Zhengqiang Pan, Narayanaswamy Balakrishnan, Quan Sun, and Jinglun Zhou. Bivariate degradation analysis of products based on wiener processes and copulas. Journal of Statistical Computation and Simulation, 83(7):1316-1329, 2013.

Chien-Yu Peng and Sheng-Tsaing Tseng. Mis-specification analysis of linear degradation models. IEEE Transactions on Reliability, 58(3):444-455, 2009.

Hao Peng, David W Coit, and Qianmei Feng. Component reliability criticality or importance measures for systems with degrading components. IEEE Transactions on Reliability, 61(1): 4-12, 2012.

Rui Peng, YF Li, WJ Zhang, and QP Hu. Testing effort dependent software reliability model for imperfect debugging process considering both detection and correction. Reliability Engineering \& System Safety, 126:37-43, 2014.

Jinlei Qin and Zheng Li. Reliability and sensitivity analysis method for a multistate system with common cause failure. Complexity, 2019. doi: 10.1155/2019/6535726.

Jinlei Qin and Zheng Li. Reliability modeling for multistate system with preventive maintenance under customer demand. Complexity, 2020. doi: 10.1155/2020/3165230.

Nipat Rasmekomen and Ajith Kumar Parlikad. Condition-based maintenance of multi-component systems with degradation state-rate interactions. Reliability Engineering \& System Safety, 148: $1-10,2016$.

Héctor Rivera-Gómez, Ali Gharbi, Jean-Pierre Kenné, Oscar Montaño-Arango, and Eva Selene Hernández-Gress. Subcontracting strategies with production and maintenance policies for a manufacturing system subject to progressive deterioration. International Journal of Production Economics, 200:103-118, 2018.

Biswajit Sarkar and Sharmila Saren. Product inspection policy for an imperfect production system with inspection errors and warranty cost. European Journal of Operational Research, 248(1): 263-271, 2016. 
Jingyuan Shen, Alaa Elwany, and Lirong Cui. Reliability analysis for multi-component systems with degradation interaction and categorized shocks. Applied Mathematical Modelling, 56:487500, 2018.

Haithem Skima, Kamal Medjaher, Christophe Varnier, Eugen Dedu, and Julien Bourgeois. A hybrid prognostics approach for mems: From real measurements to remaining useful life estimation. Microelectronics Reliability, 65:79-88, 2016.

Sanling Song, David W Coit, Qianmei Feng, and Hao Peng. Reliability analysis for multicomponent systems subject to multiple dependent competing failure processes. IEEE Transactions on Reliability, 63(1):331-345, 2014.

Chiel D Van Oosterom, Alaa H Elwany, Dilay Çelebi, and Geert-Jan Van Houtum. Optimal policies for a delay time model with postponed replacement. European Journal of Operational Research, 232(1):186-197, 2014.

CB Von der Ohe, R Johnsen, and N Espallargas. Multi-degradation behavior of austenitic and super duplex stainless steel-the effect of 4-point static and cyclic bending applied to a simulated seawater tribocorrosion system. Wear, 288:39-53, 2012.

Hai-Canh Vu, Phuc Do, and Anne Barros. A study on the impacts of maintenance duration on dynamic grouping modeling and optimization of multicomponent systems. IEEE Transactions on Reliability, 2018.

Xiaolin Wang, Narayanaswamy Balakrishnan, and Bo Guo. Residual life estimation based on a generalized wiener degradation process. Reliability Engineering \& System Safety, 124:13-23, 2014.

Yaping Wang and Hoang Pham. Modeling the dependent competing risks with multiple degradation processes and random shock using time-varying copulas. IEEE Transactions on Reliability, 61(1):13-22, 2012.

George Alex Whitmore and Fred Schenkelberg. Modelling accelerated degradation data using wiener diffusion with a time scale transformation. Lifetime Data Analysis, 3(1):27-45, 1997.

Shaomin Wu, Yi Chen, Qingtai Wu, and Zhonglai Wang. Linking component importance to optimisation of preventive maintenance policy. Reliability Engineering \& System Safety, 146:26-32, 2016. 
Yisha Xiang, David W Coit, and Qianmei Feng. Accelerated burn-in and condition-based maintenance for n-subpopulations subject to stochastic degradation. IIE Transactions, 46(10):1093$1106,2014$.

Haiyan Xu, Xiaoping Li, Rubén Ruiz, and Haihong Zhu. Group scheduling with nonperiodical maintenance and deteriorating effects. IEEE Transactions on Systems, Man, and Cybernetics: Systems, 2019.

Moon Hee Yang and Jae Hyung Cho. Minimisation of inspection and rework cost in a blu factory considering imperfect inspection. International Journal of Production Research, 52(2):384-396, 2014.

Zhenggeng Ye, Zhiqiang Cai, Fuli Zhou, Jiangbin Zhao, and Pan Zhang. Reliability analysis for series manufacturing system with imperfect inspection considering the interaction between quality and degradation. Reliability Engineering \& System Safety, 189:345-356, 2019.

Zhi-Sheng Ye, Yu Wang, Kwok-Leung Tsui, and Michael Pecht. Degradation data analysis using wiener processes with measurement errors. IEEE Transactions on Reliability, 62(4):772-780, 2013.

Uwe Zerbst, Katrin Mädler, and Hartmut Hintze. Fracture mechanics in railway applications_-an overview. Engineering Fracture Mechanics, 72(2):163-194, 2005.

Qingqing Zhai and Zhi-Sheng Ye. Rul prediction of deteriorating products using an adaptive wiener process model. IEEE Transactions on Industrial Informatics, 13(6):2911-2921, 2017.

Fengxia Zhang, Jingyuan Shen, and Yizhong Ma. Optimal maintenance policy considering imperfect repairs and non-constant probabilities of inspection errors. Reliability Engineering \& System Safety, 193:106615, 2020.

Xiujie Zhao, Jianyu $\mathrm{Xu}$, and Bin Liu. Accelerated degradation tests planning with competing failure modes. IEEE Transactions on Reliability, 67(1):142-155, 2018.

Xuejing Zhao, Mitra Fouladirad, Christophe Bérenguer, and Laurent Bordes. Condition-based inspection/replacement policies for non-monotone deteriorating systems with environmental covariates. Reliability Engineering \& System Safety, 95(8):921-934, 2010.

Deqiang Zheng and Xiangzhong Fang. Exact confidence limits for the acceleration factor under constant-stress partially accelerated life tests with type-i censoring. IEEE Transactions on Reliability, 67(1):92-104, 2018. 


\section{Appendix}

\section{(1) Proof of Proposition 1}

$K_{1}$ can be rewritten as

$$
\frac{T_{f}}{T}-1<K_{1}=\left\lfloor\frac{T_{f}}{T}\right\rfloor<\frac{T_{f}}{T} .
$$

According to model assumptions, it is easy to obtain that $K_{2}$ is inversely related to the number of failures at inspection. The more failure features exist, the easier that a failure will be detected, which leads to a smaller $K_{2}$. Since there are $m$ degradation processes, the number of existing failure features after occurrence of a failure is within the range $1 \leq N_{f} \leq m$. For any given number of existing failures $j, K_{2}$ follows a geometric distribution with the parameter $1-\beta^{j}$. The boundary of $E\left[K_{2}\right]$ can be obtained by relaxing the number of existing failure features (whether it be timevariant or not) to 1 and $m$ respectively. It follows that

$$
\frac{1}{1-\beta^{m}} \leq E\left[K_{2}\right] \leq \frac{1}{1-\beta}
$$

Combining the above two equations, we have

$$
E\left[T_{f}\right]+\frac{\beta^{m} T}{1-\beta^{m}}<E\left[K_{1}+K_{2}\right] \cdot T<E\left[T_{f}\right]+\frac{T}{1-\beta} .
$$

By substituting the above equation into Eq. (14), we can have that $C R(T)$ is limited within the range

$$
C_{d}+\frac{C_{I}}{T}+\frac{C_{R}-C_{d} E\left[T_{f}\right]}{E\left[T_{f}\right]+\frac{\beta^{m} T}{1-\beta^{m}}}<C R(T)<C_{d}+\frac{C_{I}}{T}+\frac{C_{R}-C_{d} E\left[T_{f}\right]}{E\left[T_{f}\right]+\frac{T}{1-\beta}} .
$$

\section{(2) Proof of Proposition 2}

As the first step, we need to prove that $f_{1}(T)$ and $f_{2}(T)$ are unimodal with respect to the inspection interval $T$. To do so, rewrite $f_{2}(T)$ as

$$
f_{2}(T)=C_{d}+\frac{a}{T}-\frac{b}{c T+d}
$$

where $a=C_{I}, b=C_{d} E\left[T_{f}\right]-C_{R}, c=\frac{1}{1-\beta}$, and $d=E\left[T_{f}\right]$. The derivative of $f_{2}(T)$ is given as

$$
f_{2}^{\prime}(T)=-\frac{a}{T^{2}}+\frac{b c}{(c T+d)^{2}}
$$




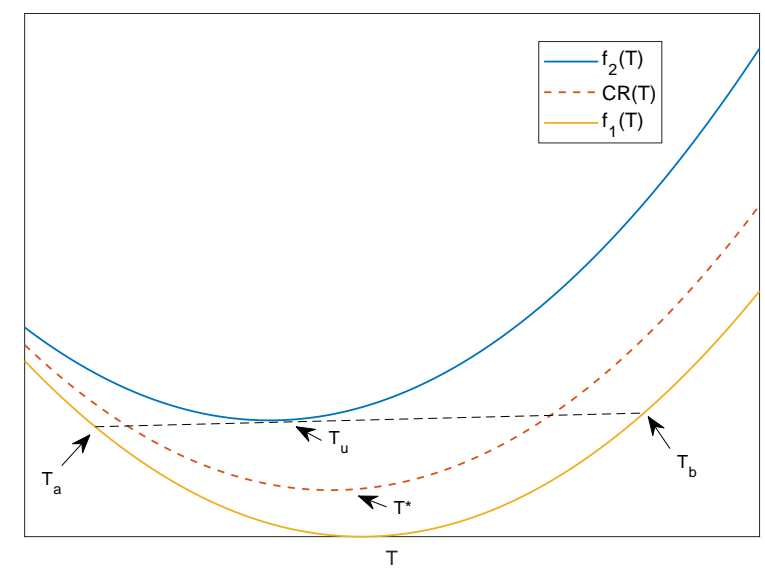

Figure 15: Illustration of Proposition 2

From $f_{2}^{\prime}(T)=0$, we have

$$
\left(b c-a c^{2}\right) T^{2}-2 a c d T-a d^{2}=0 .
$$

According to the assumption that $b>a c$, by solving the above equation, it follows that only one root exists over the range $(0,+\infty)$. Denote by $T_{u}$ the argmi point of $f_{2}(t)$. As $\lim _{T \rightarrow 0} f_{2}(T) \rightarrow+\infty$, we know that for $T \in\left(0, T_{u}\right), f_{2}(T)$ is monotonously decreasing, while for $T \in\left(T_{u},+\infty\right), f_{2}(T)$ increases monotonously. Thus, we can conclude that $f_{2}(T)$ is a unimodal function for $T \in\left(0, T_{u}\right)$. Similarly, we can conclude $f_{1}(T)$ is a unimodal function for $T \in\left(0, T_{u}\right)$.

Before we reach the final conclusion, we first need to prove that $T_{a}$ and $T_{b}$ do exist. Since it holds that $f_{1}(T)<f_{2}(T), \forall T \in(0,+\infty)$, we can have $f_{1}^{*}<f_{2}^{*}$. In addition, it holds $\lim _{T \rightarrow 0} f_{1}(T)>f_{2}^{*}$ and $\lim _{T \rightarrow \infty} f_{1}(T)=C_{d}>f_{2}^{*}$. Along with the unimodality of $f_{1}(T)$, we can conclude that there exist $T_{a}$ and $T_{b}$ that satisfy $T_{a}, T_{b} \in\left\{T: f_{1}(T)=f_{2}^{*}\right\}$. Denote $T_{u}$ as the minimum of $f_{2}(T)$, i.e., $T=T_{u}$. The conclusion can be proved by contradiction. Suppose that $T^{*}$ were no less than $T_{b}, T^{*} \geq T_{b}$, it follows that

$$
C R\left(T^{*}\right)>f_{1}\left(T^{*}\right) \geq f_{1}\left(T_{b}\right)=f_{2}^{*}
$$

However, in the previous discussion, we have

$$
C R\left(T^{*}\right) \leq C R\left(T_{u}\right)<f_{2}\left(T_{u}\right)=f_{2}^{*}
$$

which is a contradiction. Hence, we can conclude that $T^{*}<T_{b}$. Likewise, it can be concluded that $T^{*}>T_{a}$, which completes the proof. In addition, we depict the functions in Fig. 15 to better illustrate the proof of Proposition 2. 


\section{(3) Parameter estimation based on MLE}

Let

$$
\Delta \tau_{j}=t_{j+1}^{\gamma}-t_{j}^{\gamma}
$$

and

$$
\Delta \mathbf{X}\left(t_{j}\right)=\mathbf{X}\left(t_{j+1}\right)-\mathbf{X}\left(t_{j}\right)
$$

where $t_{j}$ is the $j$ th measurement time. According to the independent increment property of Wiener process, the increased degradation amount $\Delta \mathbf{X}\left(t_{j}\right)$ follows a bivariate normal distribution, i.e.,

$$
\Delta \mathbf{X}\left(t_{j}\right) \sim \mathscr{N}\left(\lambda \Delta \tau_{j}, \Delta \tau_{j} \Sigma\right)
$$

Denote $\Delta x_{1, i}^{j}$ as the increased degradation amount at $j$ th measurement time for the $i$ th sample, the log-likelihood function is given as

$$
\begin{aligned}
l(\theta) & =-\sum_{i}^{N} \sum_{j}^{M}\left[\log (2 \pi)+\log \left(\Delta \tau_{j}\right)\right. \\
& \left.+\frac{1}{2} \log (|\Xi|)+\frac{1}{2 \Delta \tau_{j}}\left(U_{i}^{j}\right)^{T} \Xi^{-1} U_{i}^{j}\right]
\end{aligned}
$$

where $N$ is the sample size, $M$ is the number of intervals of a sample, $\theta$ is the set of parameters for estimation, and

$$
U_{i}^{j}=\left[\begin{array}{c}
\Delta x_{1, i}^{j}-\lambda_{1} \Delta \tau_{j} \\
\Delta x_{2, i}^{j}-\lambda_{2} \Delta \tau_{j}
\end{array}\right] .
$$

By taking derivative with respect to $\lambda_{1}$ and $\lambda_{2}$, we can get the estimate of $\lambda_{1}$ and $\lambda_{2}$ as

$$
\tilde{\lambda}_{k}=\frac{\sum_{i}^{N} \sum_{j}^{M} \Delta x_{k, j}^{i}}{N \sum_{j}^{M} \Delta \tau_{j}}, k=1,2 .
$$

No closed form can be derived to estimate parameters of the variance matrix. Hence, we turn to numerical methods. Matlab function fminunc is used to optimize the likelihood function for the variance matrix parameters.

\section{(4) Comparison with a bivariate gamma process}

To compare with gamma processes, we first provide probability plots for Crack A and B respectively, with respect to the increments of the crack size. Figure 16 shows that both gamma and Wiener 

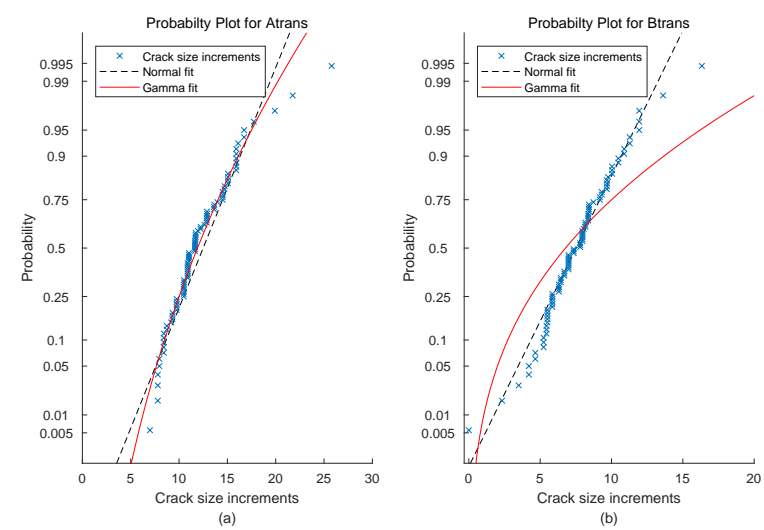

Figure 16: Probability plots for comparison of gamma and Wiener process: (a) Crack A and (b) Crack B

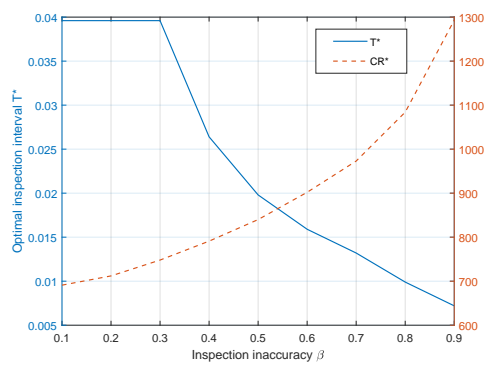

Figure 17: Variability of $C R\left(T^{*}\right)$ and $T^{*}$ vs $\beta$ for a bivariate gamma process

process fit the data well for Crack A. However, for Crack B, Wiener process performs much better than gamma process.

In addition, we compare with a bivariate gamma process to show the applicability of our model. The bivariate gamma process is constructed as follows: Let $Z_{1}(t), Z_{2}(t)$, and $Z_{u}(t)$ be three independent univariate gamma processes, with parameters $\left(\alpha_{1}, b\right),\left(\alpha_{2}, b\right)$, and $\left(\alpha_{u}, b\right)$. Define $Y_{1}(t)=Z_{1}(t)+Z_{u}(t)$, and $Y_{2}(t)=Z_{2}(t)+Z_{u}(t)$. The process $Y(t)=\left(Y_{1}(t), Y_{2}(t)\right)$ is then a bivariate subordinator whose marginal process is gamma process with parameters $\left(a_{i}, b\right)$, where $a_{i}=\alpha_{i}+\alpha_{u}$, $i=1,2$. In this way, the correlation coefficient between the two degradation processes is

$$
\rho=\frac{\alpha_{u}}{\sqrt{a_{1} a_{2}}}
$$

The parameters are set as $\left(a_{1}, a_{2}, b, \rho\right)=(12.2178,7.5967,1,0.1732)$. Figure 17 shows the variety of $C R\left(T^{*}\right)$ and $T^{*}$ with respect to $\beta$. One can observe that $C R\left(T^{*}\right)$ is increasing while $T^{*}$ presents a non-increasing trend with $\beta$. The result of Figure 17 is consistent with that in Figure 10, implying the applicability of our model for gamma processes. 


\section{(5) Derivation of Fisher information}

The Fisher information can be evaluated by

$$
I(\theta)=\left[\begin{array}{ccccc}
E_{11} & E_{12} & 0 & 0 & 0 \\
E_{21} & E_{22} & 0 & 0 & 0 \\
0 & 0 & E_{33} & E_{34} & E_{35} \\
0 & 0 & E_{43} & E_{44} & E_{45} \\
0 & 0 & E_{53} & E_{54} & E_{55}
\end{array}\right]
$$

where

$$
\begin{aligned}
& E_{11}=E\left[-\frac{\partial^{2} l(\theta)}{\partial \lambda_{1}^{2}}\right]=N \sum_{j=1}^{M} \frac{\Delta \tau_{j}}{\left(1-\rho^{2}\right) \sigma_{1}^{2}} \\
& E_{12}=E_{21}=E\left[-\frac{\partial^{2} l(\theta)}{\partial \lambda_{1} \partial \lambda_{2}}\right]=N \sum_{j=1}^{M} \frac{\rho \Delta \tau_{j}}{\left(1-\rho^{2}\right) \sigma_{1} \sigma_{2}} \\
& E_{22}=E\left[-\frac{\partial^{2} l(\theta)}{\partial \lambda_{2}^{2}}\right]=N \sum_{j=1}^{M} \frac{\Delta \tau_{j}}{\left(1-\rho^{2}\right) \sigma_{2}^{2}} \\
& E_{33}=E\left[-\frac{\partial^{2} l(\theta)}{\partial \sigma_{1}^{2}}\right]=N M \frac{2-\rho^{2}}{\left(1-\rho^{2}\right) \sigma_{1}^{2}} \\
& E_{34}=E_{43}=E\left[-\frac{\partial^{2} l(\theta)}{\partial \sigma_{1} \partial \sigma_{2}}\right]=-N M \frac{\rho^{2}}{\left(1-\rho^{2}\right) \sigma_{1} \sigma_{2}} \\
& E_{35}=E_{53}=E\left[-\frac{\partial^{2} l(\theta)}{\partial \sigma_{1} \partial \rho}\right]=-N M \frac{\rho}{\left(1-\rho^{2}\right) \sigma_{1}} \\
& E_{44}=E\left[-\frac{\partial^{2} l(\theta)}{\partial \sigma_{2}^{2}}\right]=N M \frac{2-\rho^{2}}{\left(1-\rho^{2}\right) \sigma_{2}^{2}} \\
& E_{45}=E_{54}=E\left[-\frac{\partial^{2} l(\theta)}{\partial \sigma_{2} \partial \rho}\right]=-N M \frac{\rho}{\left(1-\rho^{2}\right) \sigma_{2}} \\
& E_{55}=E\left[-\frac{\partial^{2} l(\theta)}{\partial \rho^{2}}\right]=N M \frac{1+\rho^{2}}{\left(1-\rho^{2}\right)^{2}}
\end{aligned}
$$

